

\title{
A FRONT-TRACKING SHOCK-CAPTURING METHOD FOR TWO GASES
}

\author{
Mehdi VAhaB AND GREgory H. Miller
}

\begin{abstract}
We present a new high-order front-tracking method for hyperbolic systems of conservation laws for two gases separated by a tracked contact discontinuity, using a combination of a high-order Godunov algorithm and level set methods. Our approach discretizes the moving front and gas domains on a Cartesian grid, with control volumes determined by the intersection of the grid with the front. In cut cells, a combination of conservative and nonconservative finite volume quadratures provide small-cell stability. Global conservation is maintained using redistribution. We demonstrate second-order convergence in smooth flow and first-order convergence in the presence of shocks.
\end{abstract}

\section{Introduction}

The predictive simulation of two-fluid flows with sharp material interfaces is necessary to understand processes such as shock-induced mixing that are important in ballistic impact and inertial confinement processes. Numerical methods that address sharp interfaces using discrete step functions are zero-order accurate in capturing interface jumps and cannot detect jump-sensitive features such as the onset of instability [42]. To address such problems while properly resolving jumps we present a new 2-D front-tracking method for hyperbolic systems of conservation laws specialized to gas dynamics.

Any such method may be distinguished by its front representation and the way it handles gas volume geometric calculations (see [24] for an early review of front tracking approaches). One class of front-tracking methods is surface tracking $[13 ; 17 ; 19]$. While highly successful, complex schemes are involved for front entanglement and difficulties could arise for generalization to higher dimensions. For instance, the topology of the solution to Riemann problem is not known for the general case [32]. Volume-tracking methods introduce a simpler front representation, but lack subgrid scale resolution [22]. Interface reconstruction methods have been

MSC2010: 65D32, 76T99, 35L04.

Keywords: sharp interface, front-tracking, finite-volume, multifluids, irregular geometries, Cartesian grids, shock-capturing. 
used to extract geometrical information from volume-tracking methods [22; 41]. With the introduction of level set methods, new front-tracking schemes have been developed for high-order implicit front evolution [15; 44]. Our approach is based on a new interface reconstruction method that extracts accurate geometry information from a time sequence of discrete level set data [30;36].

Depending on the representation of the front, finite volume and finite difference methods have developed different approaches to calculate state variables and fluxes near and on the front. Since the material interface moves, there may be small fractional cells near the interface. The small volume of those cells force severe limitations on unmodified finite volume and finite difference methods. Several approaches have been developed to resolve or circumvent this problem, such as cell merging [18], the h-box method [4], the ghost fluid method [15], and the single phase approximation $[8 ; 38]$. Another approach is the hybrid conservative method of Chern and Colella [7] and Bell et al. [2], which combines the conservative finite volume method with a nonconservative but stable update, and maintains global conservation using a redistribution algorithm. This idea has been successfully used for embedded boundary methods for static [11] and time-dependent $[35 ; 36$; 37] domains, and for a second-order conservative front-tracking method in one dimension [16].

We base our approach on the hybrid conservative finite volume method of Chern and Colella [7] and Pember et al. [45], which solves single phase dynamics using an unsplit Godunov method in irregular domains. The time-dependence of the domain uses space-time finite volume quadratures, generalizing the 1-D algorithm of Gatti-Bono et al. [16]. The geometric quantities that support these quadratures are derived from level sets using algorithms presented by Ligocki et al. [30] and Miller and Trebotich [36]. The material interface is indicated by the zero of a level set, which is advanced in time using well-established algorithms [14; 25]. Likewise, the extended velocity field that governs the level set evolution uses a well-established method [1] to extend a velocity computed from two-gas Riemann problems across the front. In the following technical description of the algorithm, we assume that the reader is familiar with the high-order Godunov algorithm [7; 45] and the PDE-based level set algorithms $[1 ; 14 ; 25 ; 46]$ that we employ essentially as they appear in the literature. Here the emphasis will be placed on the interconnectivity of these algorithmic components, and new specific details of implementation. We have implemented our algorithm in two spatial dimensions. The algorithm description is presented in a more general form, and we believe its implementation in 3-D will be straight-forward.

Our approach results in a robust high-order front-tracking method. Using convergence tests, we demonstrate that our method is second-order accurate for smooth flow, and first-order accurate in the presence of shocks. 


\section{Governing equations}

2.1. Gas dynamics. We are developing a hybrid numerical method for a hyperbolic system of conservation laws:

$$
\begin{aligned}
& \frac{\partial \boldsymbol{U}}{\partial t}+\nabla \cdot \overrightarrow{\boldsymbol{F}}=0, \\
& \boldsymbol{U}=\boldsymbol{U}(\boldsymbol{x}, t), \quad \boldsymbol{x} \in \boldsymbol{\Omega} \subset \mathbb{R}^{D}, \\
& \overrightarrow{\boldsymbol{F}}=\left(\boldsymbol{F}^{1} \ldots \boldsymbol{F}^{D}\right)=\overrightarrow{\boldsymbol{F}}(\boldsymbol{U}), \\
& \boldsymbol{U}, \boldsymbol{F}^{d} \in \mathbb{R}^{m},
\end{aligned}
$$

where $\boldsymbol{U}$ is a vector of conserved variables, and $\overrightarrow{\boldsymbol{F}}$ is the corresponding flux vector defined in the problem domain $\boldsymbol{\Omega}$. A front $\mathcal{F}(t)$ separates the problem domain into two subdomains $\boldsymbol{\Omega}_{1}(t)$ and $\boldsymbol{\Omega}_{2}(t)$. Each gas is governed by the equation system stated above while fulfilling the Rankine-Hugoniot jump condition on the front,

$$
\left(\overrightarrow{\boldsymbol{F}}_{1}-\overrightarrow{\boldsymbol{F}}_{2}\right) \cdot \vec{n}_{s}-s^{f}\left(\boldsymbol{U}_{1}-\boldsymbol{U}_{2}\right)=0
$$

where $\vec{n}_{s}$ is the spatial normal vector on the front from $\boldsymbol{\Omega}_{1}$ to $\boldsymbol{\Omega}_{2}$, and $s^{f}$ is the front velocity in the direction of $\vec{n}_{s}$ (Figure 1).

We restrict the presentation here to the 2-D Euler equations:

$$
\begin{aligned}
\boldsymbol{U} & =(\rho, \rho u, \rho v, E)^{T}, \\
\boldsymbol{F}^{1} & =\left(\rho u, \rho u^{2}+p, \rho u v,(E+p) u\right)^{T}, \\
\boldsymbol{F}^{2} & =\left(\rho v, \rho u v, \rho v^{2}+p,(E+p) v\right)^{T},
\end{aligned}
$$

where $\rho$ is the gas density, $u$ and $v$ are the velocities in the $x$ and $y$ direction respectively, $p$ is the pressure, and $E$ is the total energy defined by the equation of

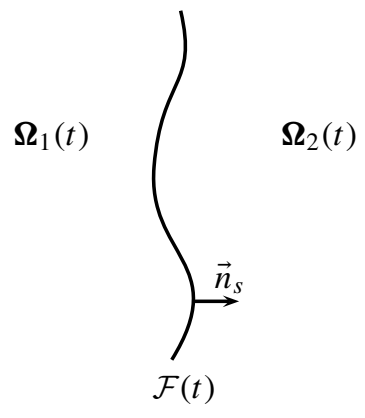

Figure 1. Two-gas flow domain and the moving interface. 
state for an ideal polytropic gas,

$$
E \equiv \frac{p}{\gamma-1}+\frac{1}{2} \rho\left(u^{2}+v^{2}\right)
$$

where $\gamma$ is the ratio of specific heats, and may be different for each gas. We use primitive variables $\boldsymbol{W}=(\rho, u, v, p)^{T}$ for flux calculations. The equation system in primitive variables is

$$
\frac{\partial \boldsymbol{W}}{\partial t}+\sum_{d=1}^{D} \boldsymbol{A}^{d} \frac{\partial \boldsymbol{W}}{\partial x_{d}}=0
$$

with

$$
\boldsymbol{A}^{1}=\left(\begin{array}{cccc}
u & \rho & 0 & 0 \\
0 & u & 0 & \rho^{-1} \\
0 & 0 & u & 0 \\
0 & \gamma p & 0 & u
\end{array}\right), \quad \boldsymbol{A}^{2}=\left(\begin{array}{cccc}
v & 0 & \rho & 0 \\
0 & v & 0 & 0 \\
0 & 0 & v & \rho^{-1} \\
0 & 0 & \gamma p & v
\end{array}\right),
$$

for $D=2$.

2.2. Front dynamics. The level set method was introduced by Osher and Sethian to implicitly evolve an interface [44] (see the books by Sethian [52], and Osher and Fedkiw [43] for a complete introduction). We use a level set function to implicitly represent the front. The level set $\phi(\vec{x}, t)$ is a continuous function with

$$
\begin{cases}\phi(\vec{x}, t)<0 & \text { in } \boldsymbol{\Omega}_{1}(t), \\ \phi(\vec{x}, t)=0 & \text { on } \mathcal{F}(t), \\ \phi(\vec{x}, t)>0 & \text { in } \boldsymbol{\Omega}_{2}(t) .\end{cases}
$$

The interface is represented by the zero level set $\mathcal{F}(t)=\{\vec{x} \mid \phi(\vec{x}, t)=0\}$. The level set is updated using the level set equation [44],

$$
\phi_{t}+\vec{v}_{\mathrm{ext}} \cdot \nabla \phi=0
$$

where $\vec{v}_{\text {ext }}$ is the extended velocity defined in $\mathbb{R}^{D}$. The extended velocity represents the movement of the whole level set function and is defined to match the velocity of the front,

$$
\vec{v}_{\text {ext }}=\vec{v}^{f} \quad \text { on } \mathcal{F}(t)
$$

The level set function $\phi$ is used for calculating accurate geometric features of the front. Therefore, it is helpful to define and maintain it as a smooth function. It is initialized as a signed distance function that satisfies (12),

$$
\phi(\vec{x}, 0)= \pm l(\vec{x}),
$$

where $l$ is the distance of point $\vec{x}$ to the front $\mathcal{F}(0)$. The level set equation (13) moves the zero level set correctly, but with time it will cease to be a distance 
function, i.e., with $|\nabla \phi|=1$. We maintain $\phi$ as a distance function by carefully generating the extended velocity [1], and by using a redistancing procedure [14]. The details are described in Section 4.3 and Section 4.4.

\section{Discretization}

3.1. Control volumes, quadrature points, and special notation. The physical problem domain is discretized with a Cartesian grid with size $h$ in space and $\Delta t$ in time. That is, a Cartesian (or regular) cell $\boldsymbol{i}$ is defined as $\Upsilon_{\boldsymbol{i}}=[\boldsymbol{i} h,(\boldsymbol{i}+\boldsymbol{\Delta}) h], \boldsymbol{i} \in \mathbb{Z}^{D}$, where $\Delta$ is a vector of ones. Let $T=\left[t^{n}, t^{n+1}\right]$ be the time interval. The spatial and space-time control volumes are then defined as

$$
\begin{aligned}
V_{i, \alpha}(t) & =\Upsilon_{i} \cap \boldsymbol{\Omega}_{\alpha}(t), \\
C_{i, \alpha} & =\left\{\vec{x} \in \mathbb{R}^{D}, t \in T \mid \vec{x} \in V_{i, \alpha}(t)\right\} .
\end{aligned}
$$

A regular space-time control volume is a rectangular cube in $\mathbb{R}^{D} \times T$ with $2 D$ faces in $\mathbb{R}^{D-1} \times T$. The center of the cell $\Upsilon_{i}$ at time $t^{n}$ is $\left(\vec{x}_{i}, t^{n}\right)$. The faces are shown by $A_{i \pm \frac{1}{2} e^{d}}$ where $\boldsymbol{e}^{d}$ is the unit vector in direction $d$ and sign \pm indicates that the face located on lower or higher side in direction $d$. Face centers are located at $\left(\vec{x}_{i \pm \frac{1}{2} e^{d}}, t^{n+\frac{1}{2}}\right)$, and are at time $n+\frac{1}{2}$ (Figure 2b).
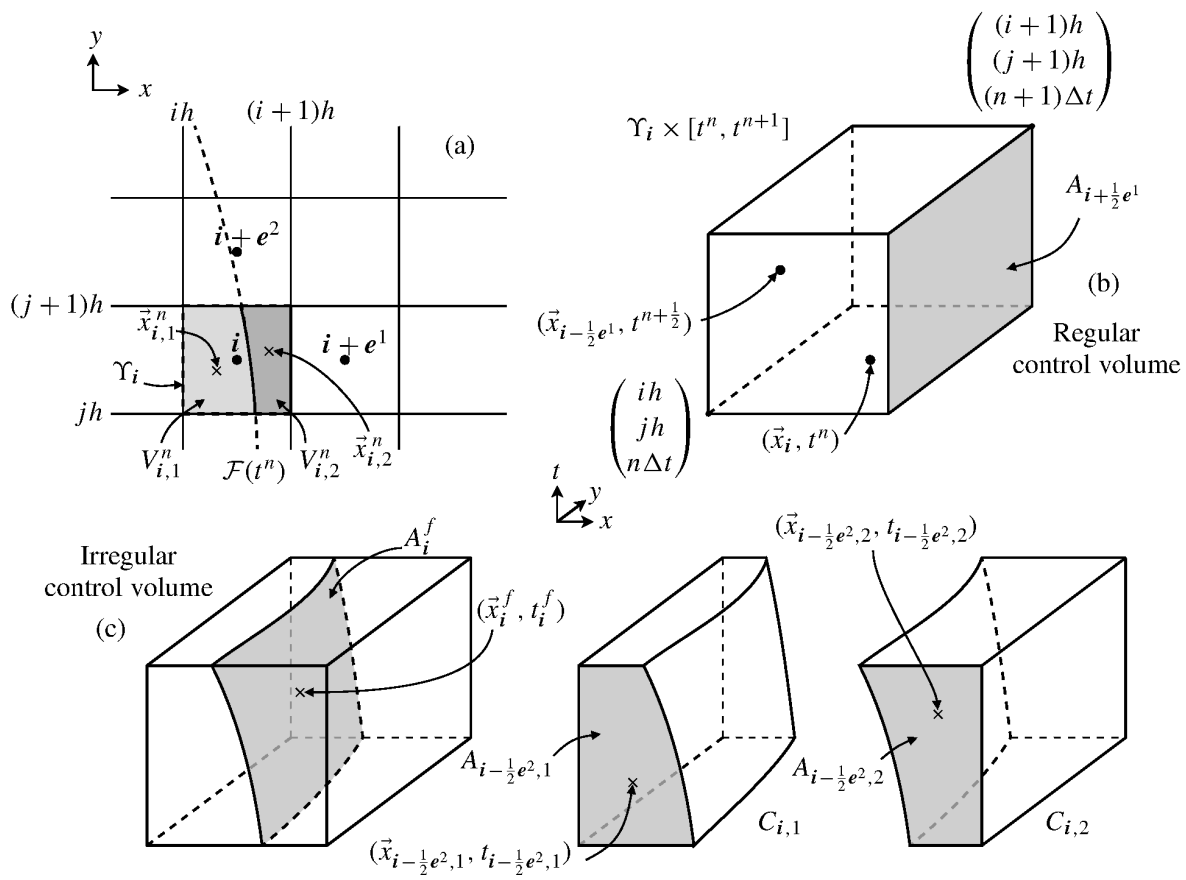

Figure 2. Geometry discretization and definitions. 
A cell, face, or volume is called irregular or cut if it intersects the front. In Figure $2 \mathrm{a}, V_{i, 1}^{n}$ and $V_{i, 2}^{n}$ denote the irregular cells corresponding to gases 1 and 2, respectively. Since such cells are fractional, the position of the center and centroid are different. The centroid of $V_{i, \alpha}^{n}$ is located at $\left(\vec{x}_{i, \alpha}, t^{n}\right)$. For faces of an irregular control volume, if a face is coincident only with the Cartesian grid, it is denoted as $A_{\boldsymbol{i} \pm \frac{1}{2} \boldsymbol{e}^{d}, \alpha}$, and its centroid as $\left(\vec{x}_{\boldsymbol{i} \pm \frac{1}{2} \boldsymbol{e}^{d}, \alpha}, t_{\boldsymbol{i} \pm \frac{1}{2} \boldsymbol{e}^{d}, \alpha}\right)$. If a face is coincident with the front, it is written as $A_{i}^{f}$ with the centroid at $\left(\vec{x}_{i}^{f}, t_{i}^{f}\right)$ (Figure $2 \mathrm{c}$ ). Based on the above definitions, the cell and face fractions are specified as follows:

$$
\Lambda_{i, \alpha}^{n}=\frac{\left|V_{i, \alpha}^{n}\right|}{h^{D}}, \quad a_{i \pm \frac{1}{2} e^{d}, \alpha}=\frac{\left|A_{i \pm \frac{1}{2} e^{d}, \alpha}\right|}{\Delta t h^{D-1}}, \quad \text { and } a_{i}^{f}=\frac{\left|A_{i}^{f}\right|}{\Delta t h^{D-1}} .
$$

The cell-centroid $\left(\vec{x}_{i, \alpha}, t^{n}\right)$ is defined as the center of $V_{i, \alpha}^{n}$,

$$
\vec{x}_{i, \alpha}=\frac{1}{\left|V_{i, \alpha}^{n}\right|} \int_{V_{i, \alpha}^{n}} \vec{x} \mathrm{~d} V
$$

and face-centroids are given by

$$
\begin{aligned}
\left(\vec{x}_{\boldsymbol{i}+\frac{1}{2} \boldsymbol{e}^{d}, \alpha}, t_{\boldsymbol{i}+\frac{1}{2} \boldsymbol{e}^{d}, \alpha}\right) & =\frac{1}{\left|A_{\boldsymbol{i}+\frac{1}{2} \boldsymbol{e}^{d}, \alpha}\right|} \int_{A_{\boldsymbol{i}+\frac{1}{2} e^{d}, \alpha}(\vec{x}, t) \mathrm{d} A \mathrm{~d} t,} \\
\left(\vec{x}_{\boldsymbol{i}}^{f}, t_{\boldsymbol{i}}^{f}\right) & =\frac{1}{\left|A_{\boldsymbol{i}}^{f}\right|} \int_{A_{\boldsymbol{i}}^{f}}(\vec{x}, t) \mathrm{d} A \mathrm{~d} t,
\end{aligned}
$$

where $\mathrm{d} V=\mathrm{d} x^{D}$ and $\mathrm{d} A=\mathrm{d} x^{D-1}$ for $D>1$. The average space-time normal vector on the front for cell $i$ is defined as

$$
\vec{n}_{i, \alpha}=\frac{1}{\left|A_{i}^{f}\right|} \int_{A_{i}^{f}} \vec{n}_{\alpha} \mathrm{d} A \mathrm{~d} t,
$$

where $\vec{n}_{\alpha}$ is the outward normal vector for gas $\alpha$.

We will distinguish fluxes $\boldsymbol{F}$ defined on the interface, on face centers, and on face centroids, by $\boldsymbol{F}^{f}, \boldsymbol{F}^{\mathrm{cr}}$, and $\boldsymbol{F}^{\mathrm{cd}}$, respectively.

Note that the quantities related to irregular geometries, having subscript $\alpha$ or superscript $f$, are inherently time dependent variables and defined in the interval $\left[t^{n}, t^{n+1}\right]$. The notation is summarized in Table 12 .

3.2. Gas dynamics. The conservation equation may be written beginning with the integral form

$$
\int_{C_{i, \alpha}}\left(\nabla, \frac{\partial}{\partial t}\right) \cdot(\overrightarrow{\boldsymbol{F}}, \boldsymbol{U}) \mathrm{d} V \mathrm{~d} t=0 .
$$

To derive the finite volume method, the divergence theorem is applied to (23) giving

$$
\oint_{\partial C_{i, \alpha}} \vec{n}_{\alpha} \cdot(\overrightarrow{\boldsymbol{F}}, \boldsymbol{U}) \mathrm{d} A=0
$$




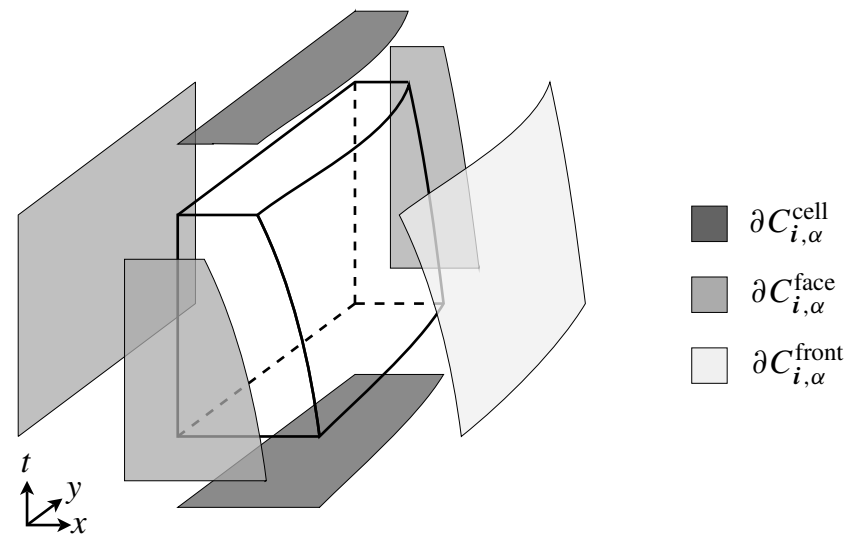

Figure 3. Separation of the integral over $\partial C_{\boldsymbol{i}, \alpha}$ based on the type of surfaces.

where $\vec{n}_{\alpha}$ and $\mathrm{d} A$ are the outward space-time normal vector and surface area differential on region $\partial C_{i, \alpha}$, respectively. Separating this integral into three parts according to surface type gives

$$
\int_{\partial C_{i, \alpha}^{\text {cell }}} \vec{n}_{\alpha} \cdot(\overrightarrow{\boldsymbol{F}}, \boldsymbol{U}) \mathrm{d} A+\int_{\partial C_{i, \alpha}^{\text {face }}} \vec{n}_{\alpha} \cdot(\overrightarrow{\boldsymbol{F}}, \boldsymbol{U}) \mathrm{d} A+\int_{\partial C_{i, \alpha}^{\text {front }}} \vec{n}_{\alpha} \cdot(\overrightarrow{\boldsymbol{F}}, \boldsymbol{U}) \mathrm{d} A=0 .
$$

The first integral is over the cells at time $t^{n}$ and $t^{n+1}$, the second integral is over the intersection of $\partial C_{i, \alpha}$ with the regular faces, and the third integral is over the intersection of $\partial C_{i, \alpha}$ with the front (Figure 3).

Discretizing in space and time gives

$$
\begin{aligned}
& \left|V_{\boldsymbol{i}, \alpha}^{n+1}\right| \boldsymbol{U}_{\boldsymbol{i}, \alpha}^{n+1}-\left|V_{\boldsymbol{i}, \alpha}^{n}\right| \boldsymbol{U}_{\boldsymbol{i}, \alpha}^{n} \\
& \quad+\sum_{ \pm, d=1}^{D}\left( \pm\left|A_{\boldsymbol{i} \pm \frac{1}{2} \boldsymbol{e}^{d}, \alpha}\right| \boldsymbol{F}_{\boldsymbol{i} \pm \frac{1}{2} \boldsymbol{e}^{d, \alpha}}^{\mathrm{cd}}\right)+\left|A_{\boldsymbol{i}}^{f}\right| \vec{n}_{\boldsymbol{i}, \alpha} \cdot\left(\overrightarrow{\boldsymbol{F}}_{\boldsymbol{i}, \alpha}^{f}, \boldsymbol{U}_{\boldsymbol{i}, \alpha}^{f}\right)=\mathcal{O}\left(h^{D+1} \Delta t\right) .
\end{aligned}
$$

Here, $\boldsymbol{U}_{\boldsymbol{i}, \alpha}^{n}$ represents the average value of $\boldsymbol{U}(\vec{x}, t)$ in $V_{\boldsymbol{i}, \alpha}^{n}$, and is centered at $\left(\vec{x}_{i, \alpha}, t^{n}\right)$, the centroid of $V_{i, \alpha}^{n}$. Flux $\boldsymbol{F}_{i \pm \frac{1}{2} e^{d}, \alpha}^{\text {cd }}$ is the average flux at the spacetime centroid of the corresponding faces in direction $d$, and $\overrightarrow{\boldsymbol{F}}_{\boldsymbol{i}, \alpha}^{f}$ and $\boldsymbol{U}_{\boldsymbol{i}, \alpha}^{f}$ are the average front flux and conservative state variable respectively, at the centroid of the front. Following the free-stream-preserving discretization of Pember et al. [45] the calculation on the front may be written in terms of fractional face areas on the 
Cartesian grid instead of relying on an estimation of the front area itself:

$$
\begin{aligned}
\left|V_{\boldsymbol{i}, \alpha}^{n+1}\right| \boldsymbol{U}_{\boldsymbol{i}, \alpha}^{n+1}-\left|V_{\boldsymbol{i}, \alpha}^{n}\right| \boldsymbol{U}_{\boldsymbol{i}, \alpha}^{n} & \\
+\sum_{ \pm, d=1}^{D}\left( \pm\left|A_{\boldsymbol{i} \pm \frac{1}{2} \boldsymbol{e}^{d}, \alpha}\right| \boldsymbol{F}_{\boldsymbol{i} \pm \frac{1}{2} \boldsymbol{e}^{d, \alpha}}^{\mathrm{d}}\right) & -\sum_{d=1}^{D}\left(\left|A_{\boldsymbol{i}+\frac{1}{2} \boldsymbol{e}^{d}, \alpha}\right|-\left|A_{\boldsymbol{i}-\frac{1}{2} \boldsymbol{e}^{d}, \alpha}\right|\right) \boldsymbol{F}_{\boldsymbol{i}, d, \alpha}^{f} \\
& -\left(\left|V_{\boldsymbol{i}, \alpha}^{n+1}\right|-\left|V_{\boldsymbol{i}, \alpha}^{n}\right|\right) \boldsymbol{U}_{\boldsymbol{i}, \alpha}^{f}=\mathcal{O}\left(h^{D+1} \Delta t\right) .
\end{aligned}
$$

Dividing by $h^{D}$ and substituting cell and face areas with unitless cell and face fractions gives

$$
\begin{aligned}
& \Lambda_{i, \alpha}^{n+1} \boldsymbol{U}_{\boldsymbol{i}, \alpha}^{n+1}-\Lambda_{i, \alpha}^{n} \boldsymbol{U}_{i, \alpha}^{n}+\frac{\Delta t}{h} \sum_{ \pm, d=1}^{D}\left( \pm a_{i \pm \frac{1}{2} \boldsymbol{e}^{d}, \alpha}\left(\boldsymbol{F}_{\boldsymbol{i} \pm \frac{1}{2} \boldsymbol{e}^{d}, \alpha}^{\mathrm{cd}}-\boldsymbol{F}_{\boldsymbol{i}, d, \alpha}^{f}\right)\right) \\
&-\left(\Lambda_{i, \alpha}^{n+1}-\Lambda_{i, \alpha}^{n}\right) \boldsymbol{U}_{\boldsymbol{i}, \alpha}^{f}=\mathcal{O}(h \Delta t) .
\end{aligned}
$$

An interim conserved variable $\boldsymbol{U}^{n, n+1}$ is defined as the conserved variable evaluated at time $t^{n}$ and centered at the cell-centroid position at time $t^{n+1}$

$$
\boldsymbol{U}_{\boldsymbol{i}, \alpha}^{n, n+1}=\boldsymbol{U}\left(\vec{x}_{\boldsymbol{i}, \alpha}\left(t^{n+1}\right), t^{n}\right),
$$

which is calculated by interpolating cell-centroid conservative variables, $\boldsymbol{U}_{i, \alpha}^{n}$, using the local grid interpolation method explained in Section 4.1. The purpose of this unusually centered variable, introduced in [16], is to make the following explicit update equation (compare (28)) uniformly centered in space:

$$
\boldsymbol{U}_{\boldsymbol{i}, \alpha}^{n+1}=\boldsymbol{U}_{\boldsymbol{i}, \alpha}^{n, n+1}-\Delta t \boldsymbol{D} \boldsymbol{F}_{\boldsymbol{i}, \alpha}^{C},
$$

where $\boldsymbol{D} \boldsymbol{F}_{\boldsymbol{i}, \alpha}^{C}$ is the conservative flux difference defined as

$$
\begin{aligned}
& \boldsymbol{D F}_{\boldsymbol{i}, \alpha}^{C}=\frac{\Lambda_{\boldsymbol{i}, \alpha}^{n+1} \boldsymbol{U}_{\boldsymbol{i}, \alpha}^{n, n+1}-\Lambda_{i, \alpha}^{n} \boldsymbol{U}_{\boldsymbol{i}, \alpha}^{n}}{\Lambda_{\boldsymbol{i}, \alpha}^{n+1} \Delta t} \\
&+\frac{1}{\Lambda_{i, \alpha}^{n+1} h}\left(\sum_{ \pm, d=1}^{D} \pm\left(a_{\boldsymbol{i} \pm \frac{1}{2} e^{d, \alpha}}\left(\boldsymbol{F}_{\boldsymbol{i} \pm \frac{1}{2} \boldsymbol{e}^{d}, \alpha}^{\mathrm{cd}}-\boldsymbol{F}_{\boldsymbol{i}, d, \alpha}^{f}\right)\right)\right. \\
&\left.\quad-\frac{h}{\Delta t}\left(\Lambda_{\boldsymbol{i}, \alpha}^{n+1}-\Lambda_{i, \alpha}^{n}\right) \boldsymbol{U}_{\boldsymbol{i}, \alpha}^{f}\right) .
\end{aligned}
$$

Note that $\boldsymbol{D} \boldsymbol{F}_{\boldsymbol{i}, \alpha}^{C}$ may become unstable for small cell fraction $\Lambda_{\boldsymbol{i}, \alpha}^{n+1}$. Therefore, a nonconservative but stable flux difference is introduced,

$$
\boldsymbol{D F}_{\boldsymbol{i}, \alpha}^{\mathrm{NC}, \mathrm{cr}}=\frac{1}{h} \sum_{ \pm, d=1}^{D} \pm \boldsymbol{F}_{i \pm \frac{1}{2} \boldsymbol{e}^{d}, \alpha}^{\mathrm{cr}},
$$


where $\boldsymbol{F}_{i \pm \frac{1}{2} e^{d}, \alpha}^{\mathrm{cr}}$ is the flux evaluated at $\left(\vec{x}_{\boldsymbol{i} \pm \frac{1}{2} e^{d}}, t^{n+\frac{1}{2}}\right)$, the center of the regular face $A_{i \pm \frac{1}{2} e^{d}}$, for gas $\alpha$. This calculation results in a cell-centered estimate of the nonconservative flux. Then, it is extrapolated to the cell-centroid position at time $t^{n+1}$, which gives $\boldsymbol{D} \boldsymbol{F}_{\boldsymbol{i}, \alpha}^{\mathrm{NC}}$. A linear combination of $\boldsymbol{D} \boldsymbol{F}_{\boldsymbol{i}, \alpha}^{C}$ and $\boldsymbol{D} \boldsymbol{F}_{\boldsymbol{i}, \alpha}^{\mathrm{NC}}$ is used for the update equation to avoid the small cell problem of the finite volume methods [10]. By picking $\Lambda_{\boldsymbol{i}, \alpha}^{n+1}$ as the linear coefficient of $\boldsymbol{D} \boldsymbol{F}_{\boldsymbol{i}, \alpha}^{C}$ a stable update method is achieved:

$$
\boldsymbol{U}_{\boldsymbol{i}, \alpha}^{n+1}=\boldsymbol{U}_{\boldsymbol{i}, \alpha}^{n, n+1}-\Delta t\left[\Lambda_{\boldsymbol{i}, \alpha}^{n+1} \boldsymbol{D} \boldsymbol{F}_{\boldsymbol{i}, \alpha}^{C}+\left(1-\Lambda_{\boldsymbol{i}, \alpha}^{n+1}\right) \boldsymbol{D} \boldsymbol{F}_{\boldsymbol{i}, \alpha}^{\mathrm{NC}}\right] .
$$

To maintain global conservation, the mass difference between the conservative update (30) and the hybrid update (33) is calculated

$$
\begin{aligned}
& \delta \boldsymbol{M}_{\boldsymbol{i}, \alpha}=\Lambda_{i, \alpha}^{n+1}\left(\left[\boldsymbol{U}_{\boldsymbol{i}, \alpha}^{n+1}-\boldsymbol{U}_{\boldsymbol{i}, \alpha}^{n, n+1}\right]_{\text {Conservative }}-\left[\boldsymbol{U}_{\boldsymbol{i}, \alpha}^{n+1}-\boldsymbol{U}_{\boldsymbol{i}, \alpha}^{n, n+1}\right]_{\text {Hybrid }}\right), \\
& \delta \boldsymbol{M}_{\boldsymbol{i}, \alpha}=\Delta t \Lambda_{\boldsymbol{i}, \alpha}^{n+1}\left(1-\Lambda_{\boldsymbol{i}, \alpha}^{n+1}\right)\left(\boldsymbol{D} \boldsymbol{F}_{\boldsymbol{i}, \alpha}^{\mathrm{NC}}-\boldsymbol{D F}_{\boldsymbol{i}, \alpha}^{C}\right),
\end{aligned}
$$

and this excess mass is redistributed to the appropriate neighbor cells of cell $\boldsymbol{i}$.

3.3. Front dynamics. The level set equation (13) is discretized in space on the same Cartesian grid using the WENO method for Hamilton-Jacobi equations by Jiang and Peng [25]. The level set function at any time step is only needed in a band around the zero level set [31]. The local level set method of Peng et al. [46] is applied to reduce the computational work for updating the level set function. This means the update method applies to the cells in a band around the zero level set. The update band should be wide enough to provide enough information to support the geometry calculation. With our choice of parameters, a $5 \times 5$ stencil of level set values is needed centered on each irregular cell.

For time discretization a two-step Adams-Bashforth method is used [34]. For the ODE system

$$
\phi_{t}=L(\phi), \quad \phi(\vec{x}, 0)=\mathcal{F}(0),
$$

the update algorithm at time step $n$ is

$$
\left\{\begin{array}{l}
\tilde{\phi}^{n+1}=\phi^{n}+\Delta t L\left(\phi^{n}\right) \\
(D \phi)^{n}=\tilde{\phi}^{n+1}-\phi^{n} \\
\phi^{n+1}=\phi^{n}+\frac{3}{2}(D \phi)^{n}-\frac{1}{2}(D \phi)^{n-1} .
\end{array}\right.
$$

The above method is second-order accurate in time with the initial starting value $(D \phi)^{-1}=(D \phi)^{0}$. For the level set update equation the $L$ operator is defined as $L(\phi)=-\vec{v}_{\text {ext }} \cdot \nabla \phi$. With this choice of time stepping, $L$ is evaluated only once per time step, which means that the two-gas front Riemann problem need be solved only once per time step to compute $\vec{v}^{f}$, which is extended to make $\vec{v}_{\text {ext }}$. 
3.4. Geometry. To calculate the update equation (33) we need the geometrical quantities defined in Section 3.1. Using $k+2$ discrete time samples of level set data $\phi^{n+1}, \phi^{n}, \ldots, \phi^{n-k}$, and applying the algorithm developed by Miller and Trebotich [36], we may evaluate the required geometry information with accuracy as high as $\mathcal{O}\left(h^{k+2}\right)$. We use $k=2$ to compute geometry information with fourth-order accuracy in our test cases. To obtain fourth-order accuracy by this approach requires a $5 \times 5$ stencil of discrete level set values at each time level.

\section{Numerical algorithms}

At the start of time step $n$ we assume that the following data are available: a centroid-centered average of the conservative variables for each gas $\boldsymbol{U}_{\boldsymbol{i}, \alpha}^{n}\left(\vec{x}_{\boldsymbol{i}, \alpha}^{n}, t^{n}\right)$; a time sequence of $k+1$ discrete level sets $\phi^{n}, \ldots, \phi^{n-k}$; the difference $(D \phi)^{n-1}$ (36); and spatial geometry information constructed from $\phi^{n}$. The goal is to find $\boldsymbol{U}_{\boldsymbol{i}, \alpha}^{n+1}\left(\vec{x}_{\boldsymbol{i}, \alpha}^{n+1}, t^{n+1}\right)$ and $\phi^{n+1}$. A brief overview of the strategy follows. Additional details are given in [57].

First, we extrapolate the conservative data from cell centroids to cell centers to support a standard high-order Godunov method. Primitive cell-centered data is extrapolated to the front centroids at time $t^{n}$ to pose Riemann problems that determine the front velocity $\vec{v}^{f}$.

The front centroid velocity is extended in a band of cells around the zero level set to make $\vec{v}_{\text {ext }}$, which is used in (36) to compute $\phi^{n+1}$ to second-order. The time sequence $\phi^{n+1}, \phi^{n}, \ldots, \phi^{n-k}$ then supports the computation of space-time geometry information in all irregular control volumes.

Following a standard high-order unsplit Godunov method, modified on irregular boundaries [9;11], we calculate the slopes and fluxes on the face and front centroids and apply the update equation (33). Excess mass is calculated and redistributed.

4.1. Local grid interpolation. The interpolation steps used in the algorithm are linear estimates based on the local grid data. If we want to interpolate the value of function $G$ at target point $\vec{x}_{t}$ based on the support data at $\vec{x}_{s}$ we write the Taylor expansion of $G\left(\vec{x}_{s}\right)$ centered at $\vec{x}_{t}$,

$$
G\left(\vec{x}_{s}\right) \approx G\left(\vec{x}_{t}\right)+\nabla G\left(\vec{x}_{t}\right)\left(\vec{x}_{s}-\vec{x}_{t}\right) .
$$

By gathering enough support point data around the target we create a least-squares problem to solve for $G\left(\vec{x}_{t}\right)$,

$$
\left(\begin{array}{cc}
1 & \left(\vec{x}_{1}-\vec{x}_{t}\right) \\
\vdots & \vdots \\
1 & \left(\vec{x}_{m}-\vec{x}_{t}\right)
\end{array}\right)\left(\begin{array}{c}
G\left(\vec{x}_{t}\right) \\
\nabla G\left(\vec{x}_{t}\right)
\end{array}\right)=\left(\begin{array}{c}
G\left(\vec{x}_{1}\right) \\
\vdots \\
G\left(\vec{x}_{m}\right)
\end{array}\right),
$$




\begin{tabular}{|l|l|l|l|l|l|l|}
\hline 9 & 8 & 7 & 6 & 7 & 8 & 9 \\
\hline 8 & 5 & 4 & 3 & 4 & 5 & 8 \\
\hline 7 & 4 & 2 & 1 & 2 & 4 & 7 \\
\hline 6 & 3 & 1 & 0 & 1 & 3 & 6 \\
\hline 7 & 4 & 2 & 1 & 2 & 4 & 7 \\
\hline 8 & 5 & 4 & 3 & 4 & 5 & 8 \\
\hline 9 & 8 & 7 & 6 & 7 & 8 & 9 \\
\hline
\end{tabular}

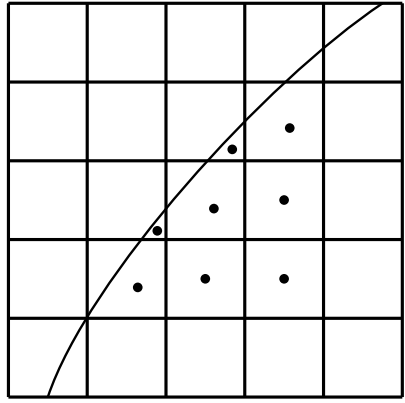

(b)

(a)

Figure 4. (a) Cell stencil showing sets of nearest neighbors. Neighbor cells with the same approximate distance to the center cell are indicated by a common number. (b) Interpolation from cell centroid data to cell center: Target point (the cell center) is shown with $\times$ and support points are shown with $\bullet$. In this example only sets 1 and 2 of nearest neighbor cells are used.

where $\vec{x}_{1}, \ldots, \vec{x}_{m}$ are support points. The support points are chosen based on the nearest cell criteria to the target cell position. We start from the most compact stencil possible and try solve the above least-square system with QR decomposition (Figure 4). More support points are added if values on the diagonal of $R$ are too small, which signals large numerical errors. The heuristic cut-off values that we used are $10^{-10}$ for $R_{i i}$ (no sum), and $10^{-15}$ for $\operatorname{det}(R)$.

4.2. Solution on the front and velocity matching. We need the state variables on the front to calculate the front speed at time $t^{n}$, and the flux on the front centroid. In both cases, the state variables from each gas are extrapolated to the front centroids. We solve the Riemann problem in front normal direction for a contact discontinuity to determine the states on the front, using a primitive variable Riemann solver [56].

The Riemann solver provides a unique normal velocity $u^{*}$, from which we determine $\vec{v}^{f}=u^{*} \mathbf{n}$. The tangential component of the front velocity is double valued, and not needed for level set advection.

When computing $\boldsymbol{D} \boldsymbol{F}^{C}(31)$ we require the flux at the space-time front centroid. This flux is computed by first setting up and solving a Riemann problem as described above with left-hand and right-hand values interpolated as described below in (48). This computation also yields a unique normal velocity $u^{*}$, however this velocity is 
not consistent with the space-time slope of the front, which gives velocity

$$
s^{f}=\frac{-n_{t}}{\sqrt{n_{x}^{2}+n_{y}^{2}}}, \quad \vec{n}_{i}^{n}=\left(n_{x}, n_{y}, n_{t}\right)^{T} .
$$

We substitute $s^{f}$ for $u^{*}$ in this Riemann problem calculation to give front values that are internally consistent and make the method fully conservative. Additional details are given in [57].

4.3. Velocity extension. We need the velocity defined on cell centers in a band around the zero level set to update the level set equation. The solution on the front at time $t^{n}$ gives the velocity on front centroids.

To keep $\phi$ a signed distance function after updating the level set equation one needs to impose the following condition on the velocity [1]:

$$
\nabla\left(\vec{v}_{\mathrm{ext}, d}\right) \cdot \nabla \phi=0, \quad d=1, \ldots, D,
$$

which means that velocity is constant along the level set gradient. We use the extension method developed by Peng et al. [46] and Zhao et al. [60]. To extend the quantity $q$ with the condition $\nabla q \cdot \nabla \phi=0$ we solve

$$
q_{t}+S(\phi) \frac{\nabla \phi}{|\nabla \phi|} \cdot \nabla q=0,
$$

as an internal boundary value problem, where $S(\phi)$ is a regularized sign function [54]. Since the characteristics of (41) are normal to the front and point outward from the front, we only need to initialize $q$ on a initialization band around $\mathcal{F}$, with a width of 2 or 3 cells, and then solve (41) while the velocity is anchored on the initialization band.

To set up the initialization band around the zero level set we pick cells that have amongst their $3^{D}-1$ neighbors at least one cell with the opposite level set sign. Applying the extension condition (40) means the extended velocity field does not change in the direction of the level set gradient. Therefore, if we project from a point outside the front, such as the center of a cell in the initialization band $\vec{x}_{c}$, in the direction of the level set gradient toward the front and reach a point on the front such as $\vec{x}_{f}$,

$$
\vec{x}_{f} \approx \vec{x}_{c}-\phi\left(\vec{x}_{c}\right) \frac{\nabla \phi\left(\vec{x}_{c}\right)}{\left|\nabla \phi\left(\vec{x}_{c}\right)\right|}
$$

(see Figure 5), then the extension condition (40) recovers

$$
\vec{v}\left(\vec{x}_{c}\right)=\vec{v}\left(\vec{x}_{f}\right) .
$$

To find the velocity at the projected point $\vec{x}_{f}$, we apply linear interpolation using the velocity values on the front centroids in the $3^{D}$ cells near $\vec{x}_{f}$. If $m$ front centroid 


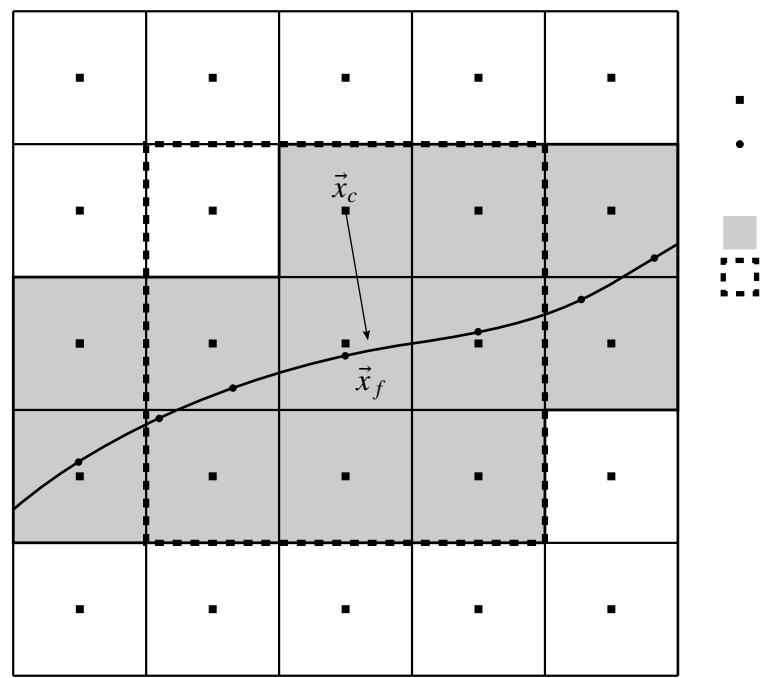

cell center

front centroid projection point initilization band interpolation area

Figure 5. Initialization of velocity field in a narrow band around zero level set.

points reside in the vicinity of $\vec{x}_{f}$, we solve the least-squares equation system (38) with $G=v_{d}\left(\vec{x}_{f}\right)$. In this case, we solve system (38) using singular value decomposition because the matrix may be singular, e.g., if the support points are collinear.

Extension equation (41) is solved iteratively as described by Peng et al. [46] to extend the velocity from the initialization band to a narrow band around the zero level set while applying the high-order WENO discretization of Jiang and Peng [25] to increase the accuracy of the algorithm and decrease the number of iterations that are needed.

4.4. Redistancing. A level set function initialized as a distance function and driven with a nonconstant velocity field may deviate from being a distance function [46; 50], causing poor evaluation of geometric quantities. Sussman et al. [54] introduced the redistancing equation in artificial time $\tau$

$$
\phi_{\tau}+S(\tilde{\phi})(|\nabla \phi|-1)=0,
$$

where $\tilde{\phi}=\phi(\vec{x}, \tau=0)$. We solve the redistancing equation (44) using the improved Hamilton-Jacobi WENO algorithm with Godunov approximation in space and the third-order TVD Runge-Kutta scheme in time by du Chéné et al. [14].

4.5. Flux calculation. There are four different types of fluxes needed to apply the hybrid update method, varying in centering location.

4.5.1. Flux on a regular face. A regular face is not intersected by the front in $\left[t^{n}, t^{n+1}\right]$. The most common flux calculated is $\boldsymbol{F}_{\boldsymbol{i} \pm \frac{1}{2} \boldsymbol{e}^{d}, \alpha}^{\mathrm{cr}}$ for a regular face, which is 
the flux on the face center $\left(\vec{x}_{\boldsymbol{i} \pm \frac{1}{2} \boldsymbol{e}^{d}}, t^{n+\frac{1}{2}}\right)$. This is done using a high-order Godunov method with transverse flux correction in primitive variables $[9 ; 11]$.

4.5.2. Flux on a covered face. If cell $\boldsymbol{i}$ is irregular, but the face at $\boldsymbol{i} \pm \frac{1}{2} \boldsymbol{e}^{d}$ does not intersect the front, then that face is said to be covered with respect to the gas $\alpha$ that is not present at $\boldsymbol{i} \pm \frac{1}{2} \boldsymbol{e}^{d}$. Fluxes on covered faces may be used in (32). The upwinding extrapolation algorithm that is used for flux calculation on a regular face only provides the state variable on one side of the covered face. A method similar to the procedure described in $[11, \S 5.2]$ is applied to extrapolate the state variables on the other side using the interior face-centered values. We use the spatial projection of $\vec{n}_{i, \alpha}$ in 2-D as the normal vector needed in the method.

4.5.3. Flux on an irregular face. A face is irregular if it intersects the front in $\left[t^{n}, t^{n+1}\right]$. We evaluate the average flux on the irregular face using the primitive states on the face centroid,

$$
\boldsymbol{F}_{\boldsymbol{i} \pm \frac{1}{2} \boldsymbol{e}^{d}, \alpha}^{\mathrm{cd}}=\boldsymbol{F}^{d}\left(\boldsymbol{W}_{\boldsymbol{i} \pm \frac{1}{2} \boldsymbol{e}^{d}, \alpha}^{\mathrm{cd}}\right) .
$$

We extrapolate the primitive variable on the face center $\boldsymbol{W}_{\boldsymbol{i} \pm \frac{1}{2} \boldsymbol{e}^{d}, \alpha}^{\mathrm{cr}}$, calculated in Section 4.5.1, to the face centroid using the average slopes of two surrounding cells in time and space,

$$
\begin{aligned}
\boldsymbol{W}_{\boldsymbol{i} \pm \frac{1}{2} \boldsymbol{e}^{d, \alpha}}^{\mathrm{cd}}=\boldsymbol{W}_{\alpha}\left(\vec{x}_{\boldsymbol{i} \pm \frac{1}{2} \boldsymbol{e}^{d}, \alpha}, t_{\boldsymbol{i} \pm \frac{1}{2} \boldsymbol{e}^{d, \alpha}}\right) & \\
=\boldsymbol{W}_{\boldsymbol{i} \pm \frac{1}{2} \boldsymbol{e}^{d}, \alpha}^{\mathrm{cr}} & +\left(\vec{x}_{\boldsymbol{i} \pm \frac{1}{2} \boldsymbol{e}^{d}, \alpha}\left(t^{n}\right)-\vec{x}_{\boldsymbol{i} \pm \frac{1}{2} \boldsymbol{e}^{d}}\right) \cdot \boldsymbol{e}^{d}\left(\frac{\Delta_{x}^{d^{\prime}} \boldsymbol{W}_{\boldsymbol{i}, \alpha}^{n}+\Delta_{x}^{d^{\prime}} \boldsymbol{W}_{\boldsymbol{i} \pm \boldsymbol{e}^{d}, \alpha}^{n}}{2 h}\right) \\
& +\left(t_{\boldsymbol{i} \pm \frac{1}{2} \boldsymbol{e}^{d}, \alpha}-\left(n+\frac{1}{2}\right) \Delta t\right)\left(\frac{\Delta_{t} \boldsymbol{W}_{\boldsymbol{i}, \alpha}^{n}+\Delta_{t} \boldsymbol{W}_{\boldsymbol{i} \pm \boldsymbol{e}^{d}, \alpha}^{n}}{2 \Delta t}\right), \quad d \neq d^{\prime},
\end{aligned}
$$

where $\Delta_{t} \boldsymbol{W}_{\boldsymbol{i}, \alpha}^{n}$ is an estimate of time difference calculated as

$$
\Delta_{t} \boldsymbol{W}_{i, \alpha}^{n}=2\left(\frac{1}{2 D} \sum_{ \pm} \sum_{d=1}^{D} \boldsymbol{W}_{\boldsymbol{i} \pm \frac{1}{2} \boldsymbol{e}^{d}, \alpha}^{\mathrm{cr}}-\boldsymbol{W}_{\boldsymbol{i}, \alpha}^{n}\right) .
$$

4.5.4. Flux on the front. The flux on the front is calculated using the solution of the Riemann problem on the contact discontinuity described in Section 4.2. The left-hand and right-hand states of that Riemann problem are derived by extrapolation from cell-centered primitive states using the local slopes in space and time for each gas,

$$
\begin{aligned}
\widetilde{\boldsymbol{W}}_{\boldsymbol{i}, \alpha}^{f} & =\widetilde{\boldsymbol{W}}_{\alpha}\left(\vec{x}_{\boldsymbol{i}}^{f}, t_{\boldsymbol{i}}^{f}\right) \\
& =\boldsymbol{W}_{\boldsymbol{i}, \alpha}^{n}+\frac{1}{h} \sum_{d=1}^{D}\left(\vec{x}_{\boldsymbol{i}}^{f}-\vec{x}_{\boldsymbol{i}, \alpha}\left(t^{n}\right)\right) \cdot \boldsymbol{e}^{d} \Delta_{x}^{d} \boldsymbol{W}_{\boldsymbol{i}, \alpha}^{n}+\frac{1}{\Delta t}\left(t_{\boldsymbol{i}}^{f}-n \Delta t\right) \Delta_{t} \boldsymbol{W}_{\boldsymbol{i}, \alpha}^{n} .
\end{aligned}
$$


The solution to the Riemann problem in the front normal direction with initial states $\widetilde{\boldsymbol{W}}_{\boldsymbol{i}, 1}^{f}$ and $\widetilde{\boldsymbol{W}}_{\boldsymbol{i}, 2}^{f}$ gives the primitive state on both sides of the front. These values, modified as described in Section 4.2, determine $\boldsymbol{W}_{\boldsymbol{i}, 1}^{f}$ and $\boldsymbol{W}_{\boldsymbol{i}, 2}^{f}$, which are used for the calculation of $\boldsymbol{F}_{\boldsymbol{i}, \alpha}^{f}$ and $\boldsymbol{U}_{\boldsymbol{i}, \alpha}^{f}$.

4.6. Excess generalized mass and redistribution. To maintain global conservation the generalized mass (i.e., $\sum_{\boldsymbol{i}}\left|V_{\boldsymbol{i}, \alpha}\right| \boldsymbol{U}_{\boldsymbol{i}, \alpha}$ ) difference between the hybrid method and the conservative method (34) should be redistributed. First we balance the excess generalized mass on the front cells based on the characteristics in the front normal coordinate systems using the approach of Chern and Colella [7]. The excess generalized mass on each side is projected on the characteristics of the cell centered values in the front normal coordinate system,

$$
\delta \boldsymbol{M}_{\boldsymbol{i}, \alpha}=\sum_{k=0}^{m-1} b_{k, \alpha} \boldsymbol{r}_{k, \alpha},
$$

where $\boldsymbol{r}_{k, \alpha}$ are the right eigenvectors of $\frac{\partial \overrightarrow{\boldsymbol{F}}}{\partial \boldsymbol{U}}\left(\boldsymbol{U}_{\boldsymbol{i}, \alpha}^{n}\right)$. Then, the contribution of excess generalized mass to each side is calculated by considering the characteristic speed of each projected term. Assuming that $\tilde{u}_{1}$ and $\tilde{c}_{1}$ are the normal velocity and sound speed of $\boldsymbol{U}_{\boldsymbol{i}, 1}^{n}$ in the front normal coordinate system, the projected components corresponding to $\tilde{u}_{1}-\tilde{c}_{1}$ and $\tilde{u}_{1}$ remain in gas 1 , and the projected component corresponding to $\tilde{u}_{1}+\tilde{c}_{1}$ moves to gas 2 . With a similar argument for gas 2 , the excess generalized mass values are:

$$
\begin{aligned}
& \delta \boldsymbol{M}_{i, 1}:=\left(b_{0,1} \boldsymbol{r}_{0,1}+b_{1,1} \boldsymbol{r}_{1,1}+b_{2,1} \boldsymbol{r}_{2,1}\right)+b_{0,2} \boldsymbol{r}_{0,2}, \\
& \delta \boldsymbol{M}_{\boldsymbol{i}, 2}:=b_{3,1} \boldsymbol{r}_{3,1}+\left(b_{1,2} \boldsymbol{r}_{1,2}+b_{2,2} \boldsymbol{r}_{2,2}+b_{3,2} \boldsymbol{r}_{3,2}\right) .
\end{aligned}
$$

Note that physical mass (i.e., $\sum_{\boldsymbol{i}}\left|V_{\boldsymbol{i}, \alpha}\right| \rho_{\boldsymbol{i}, \alpha}$ ) is not redistributed across a front since the characteristic carrying mass has velocity $\tilde{u}_{\alpha}$.

In general, the redistribution method is

$$
\boldsymbol{U}_{\boldsymbol{j}, \alpha}^{n+1}:=\boldsymbol{U}_{\boldsymbol{j}, \alpha}^{n+1}+w_{\boldsymbol{i}, \boldsymbol{j}} \delta \boldsymbol{M}_{\boldsymbol{i}, \alpha}, \quad \boldsymbol{j} \in N(\boldsymbol{i}),
$$

where $N(\boldsymbol{i})$ indicates a set of cells in the neighborhood of cell $\boldsymbol{i}$ and the weight coefficients satisfy [11],

$$
w_{i, j} \geq 0, \quad \text { and } \sum_{j \in N(i)} w_{i, j} \Lambda_{j}^{n+1}=1 .
$$

We use

$$
w_{i, j}=\frac{1}{\sum_{l \in N(i)} \Lambda_{l}^{n+1}} .
$$


With these weight coefficients we get

$$
\Lambda_{\boldsymbol{j}, \alpha}^{n+1} \boldsymbol{U}_{\boldsymbol{j}, \alpha}^{n+1}:=\Lambda_{\boldsymbol{j}, \alpha}^{n+1} \boldsymbol{U}_{\boldsymbol{j}, \alpha}^{n+1}+\frac{\Lambda_{\boldsymbol{j}, \alpha}^{n+1}}{\sum_{\boldsymbol{k} \in N(\boldsymbol{i})} \Lambda_{\boldsymbol{k}, \alpha}^{n+1}} \delta \boldsymbol{M}_{\boldsymbol{i}, \alpha}, \quad \boldsymbol{j} \in N(\boldsymbol{i}),
$$

which means the excess mass of cell $\boldsymbol{i}$ is redistributed into neighbor cells in proportion to their volume at time $n+1$.

\section{Results}

In this section we present numerical tests of the method for gas dynamics problems. The first two tests are for smooth and steady flows, where no discontinuity other than the material interface is present. The third and fourth test include the interaction of the front with a shock wave. In the fifth and sixth test cases, front dynamics are unstable by nature, and the geometry of the front is changing more than in the previous cases.

To make quantitative comparisons we define the error for conservative state variables

$$
\boldsymbol{E}_{\boldsymbol{i}, \alpha}^{2 h}=\boldsymbol{U}_{\boldsymbol{i}, \alpha}^{2 h}(t)-\boldsymbol{U}_{\boldsymbol{i}, \alpha}^{e}(t)
$$

where $\boldsymbol{U}_{\boldsymbol{i}, \alpha}^{2 h}(t)$ is calculated with spatial grid size $2 h$ and $\boldsymbol{U}_{\boldsymbol{i}, \alpha}^{e}$ is the exact solution. If we do not have the exact solution in hand, we replace it with the solution from the simulation with a finer grid. If we choose grid size $h$ for our finer grid calculation

$$
\boldsymbol{U}_{\boldsymbol{i}, \alpha}^{e}(t) \approx \frac{\sum_{\boldsymbol{j} \in M(\boldsymbol{i})} \Lambda_{\boldsymbol{j}, \alpha}^{h} \boldsymbol{U}_{\boldsymbol{j}, \alpha}^{h}(t)}{\sum_{\boldsymbol{j} \in M(\boldsymbol{i})} \Lambda_{\boldsymbol{j}, \alpha}^{h}},
$$

where $M(\boldsymbol{i})=[2 \boldsymbol{i}, 2 \boldsymbol{i}+\boldsymbol{\Delta}]$ are the fine cells underlying coarse cell $\boldsymbol{i}$.

The total error in the $L_{1}, L_{2}$ and $L_{\infty}$ norms is

$$
\begin{aligned}
\epsilon_{L_{1}}^{h} & =\sum_{\alpha, \boldsymbol{i} \in \boldsymbol{\Omega}_{\alpha}} h^{d} \Lambda_{\boldsymbol{i}, \alpha}^{h}\left|\boldsymbol{E}_{\boldsymbol{i}, \alpha}^{h}\right|, \\
\epsilon_{L_{2}}^{h} & =\left(\sum_{\alpha, \boldsymbol{i} \in \boldsymbol{\Omega}_{\alpha}} h^{d} \Lambda_{\boldsymbol{i}, \alpha}^{h}\left|\boldsymbol{E}_{\boldsymbol{i}, \alpha}^{h}\right|^{2}\right)^{\frac{1}{2}}, \\
\epsilon_{L_{\infty}}^{h} & =\max _{\alpha, \boldsymbol{i} \in \boldsymbol{\Omega}_{\alpha}}\left|\boldsymbol{E}_{\boldsymbol{i}, \alpha}^{h}\right|,
\end{aligned}
$$

and the convergence rate is calculated by

$$
p=\frac{\log \left(\epsilon^{2 h} / \epsilon^{h}\right)}{\log (2)} .
$$


5.1. Convergence test-advection. With this simple test, we examine the convergence and conservation of the method for smooth gas and front dynamics. The problem domain is a 1 by 1 square. The initial material interface is a circle with radius 0.2 centered at $(0.25,0.25)$. With the initial condition shown in Table 1 and appropriate inflow and outflow boundary conditions, simulations are done up to $t=0.5$ with CFL number 0.5 , which results in diagonal advection of the circular front without any deformation across the domain. Table 2 shows the error and convergence rates. The results show second order convergence for all norms. Fedkiw et al. [15] did a similar test and showed second order convergence in $L_{1}$. Here we also measure the convergence in $L_{\infty}$ which shows the method is second order convergent away from and near the interface.

The final front position is displayed in Figure 6 for different resolutions. Since the final solution is trivial, we measured the least squares error of the front centroid positions using the exact solution. Numerical convergence analysis shows the front position is converging with $\mathcal{O}\left(h^{1.74}\right)$ (see Figure 7).

At the request of a reviewer we measured the total amount of each conserved variable for each gas. Taking into consideration the boundary fluxes, the mass, momentum, and energy of each gas is conserved to within machine precision in each time step, and the cumulative loss of conservation is less than $10^{-16}$ for all conserved variables in all resolutions tested.

5.2. Convergence test - smooth perturbation. We test our method for a $2-\mathrm{D}$ perturbation problem to observe the interaction of the front with smooth flows. The problem domain is a 1 by 1 square. The initial front is a circle with radius 0.27 centered at $(0.5,0.5)$. The gas inside the front has a smooth bell-shaped perturbation in the pressure component of the form

$$
p_{\text {in }}(r)=p_{\text {base }}\left(1+256 \alpha\left(\frac{r+r_{p}}{2 r_{p}}-\left(\frac{r+r_{p}}{2 r_{p}}\right)^{2}\right)^{4}\right), \quad r \leq r_{p},
$$

where $p_{\text {base }}=1$ is the background value of pressure, $\alpha=0.15$ determines the peak-to-base value of the perturbation, $r_{p}=0.25$ is the radius of the perturbation and $r$ is the distance from the center of the problem domain. The initial density is

\begin{tabular}{ccc}
\hline & Gas 1: inside & Gas 2: outside \\
\hline$\rho$ & 0.138 & 1 \\
$u$ & 1 & 1 \\
$v$ & 1 & 1 \\
$p$ & 1 & 1 \\
$\gamma$ & 1.67 & 1.4 \\
\hline
\end{tabular}

Table 1. Initial values for the advection problem. 


\begin{tabular}{ccccccc}
\multicolumn{7}{c}{$\rho$} \\
\hline $\mathrm{h}$ & $L_{1}$ & rate & $L_{2}$ & rate & $L_{\infty}$ & rate \\
\hline $1 / 32$ & $5.24 \times 10^{-5}$ & & $1.84 \times 10^{-4}$ & & $4.41 \times 10^{-3}$ & \\
$1 / 64$ & $4.95 \times 10^{-6}$ & 3.40 & $2.05 \times 10^{-5}$ & 3.16 & $6.47 \times 10^{-4}$ & 2.77 \\
$1 / 128$ & $1.19 \times 10^{-6}$ & 2.06 & $3.22 \times 10^{-6}$ & 2.67 & $1.47 \times 10^{-4}$ & 2.36 \\
$1 / 256$ & $2.73 \times 10^{-7}$ & 2.12 & $7.93 \times 10^{-7}$ & 2.27 & $3.48 \times 10^{-5}$ & 2.48 \\
\hline & & & $\rho u \& \rho v$ & & & \\
\hline $\mathrm{h}$ & $L_{1}$ & rate & $L_{2}$ & rate & $L_{\infty}$ & rate \\
\hline $1 / 32$ & $1.12 \times 10^{-4}$ & & $2.73 \times 10^{-4}$ & & $4.12 \times 10^{-3}$ & \\
$1 / 64$ & $1.00 \times 10^{-5}$ & 3.49 & $3.43 \times 10^{-5}$ & 2.99 & $6.42 \times 10^{-4}$ & 2.68 \\
$1 / 128$ & $2.18 \times 10^{-6}$ & 2.20 & $8.05 \times 10^{-6}$ & 2.09 & $1.36 \times 10^{-4}$ & 2.24 \\
$1 / 256$ & $5.38 \times 10^{-7}$ & 2.02 & $1.91 \times 10^{-6}$ & 2.08 & $3.30 \times 10^{-4}$ & 2.04 \\
\hline & & & $E$ & & & \\
\hline $\mathrm{h}$ & & & & & & \\
\hline $1 / 32$ & $2.70 \times 10^{-4}$ & & $7.09 \times 10^{-4}$ & & $1.34 \times 10^{-2}$ & \\
$1 / 64$ & $2.80 \times 10^{-5}$ & 3.27 & $1.09 \times 10^{-4}$ & 2.70 & $2.45 \times 10^{-3}$ & 2.45 \\
$1 / 128$ & $6.14 \times 10^{-6}$ & 2.19 & $1.91 \times 10^{-5}$ & 2.51 & $4.77 \times 10^{-4}$ & 2.40 \\
$1 / 256$ & $1.40 \times 10^{-6}$ & 2.13 & $4.30 \times 10^{-6}$ & 2.15 & $1.11 \times 10^{-4}$ & 2.10 \\
\hline
\end{tabular}

Table 2. Error and convergence rate for the advection problem.

constant and equals 1 for both gases, and the initial velocities are zero. The initial pressure for the gas outside the front is $p_{\text {base }}$. We simulate the state variables to a fixed time $t=0.6$ to allow the perturbation to pass the front with a CFL number of 0.5 . We simulated the expansion of the perturbation in seven grid sizes varying from $\frac{1}{32}$ to $\frac{1}{2048}$, and calculated the solution errors and convergence rates based on the Richardson method explained above.

Two cases for gas materials are considered. First, gases have different ratios of specific heats, $\gamma_{\text {in }}=1.67$ and $\gamma_{\text {out }}=1.4$ (see Table 3 ). Second, both gases are the same material, $\gamma_{\text {in }}=\gamma_{\text {out }}=1.4$ (see Table 4). Our method shows second-order convergence in $L_{1}, L_{2}$ and $L_{\infty}$ for all conserved variables in either case.

In the second case, since we chose the same material for both gases, we expect to have continuous states on the front. It is also verified by comparing with a single gas simulation, where no front tracking is performed and the same initial condition is used. Figure 8 shows the final results, which also exhibit the expected rotational symmetry. 


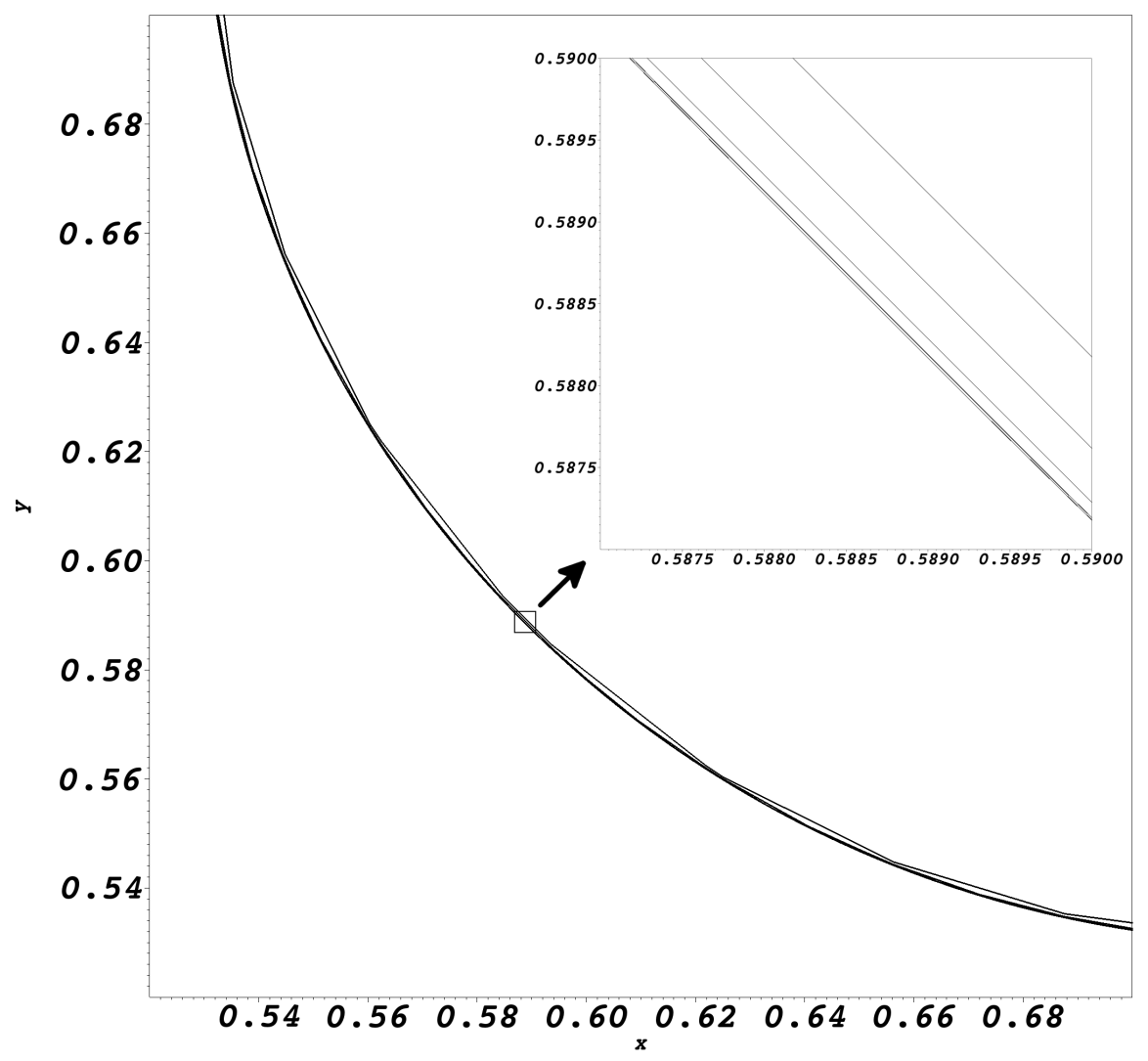

Figure 6. Final front position in the front advection problem. Inset: blowup of the front position. From right to left for $h$ equal to $\frac{1}{32}, \frac{1}{64}, \frac{1}{128}, \frac{1}{256}$ and $\frac{1}{512}$.

Using finer resolution simulations as the reference (Richardson error), we calculated the least squares error of the front centroid positions, which shows a convergence rate of $\mathcal{O}\left(h^{1.60}\right)$ (see Figure 7).

5.3. Shock test-2-D Sod problem. Sod [53] introduced this benchmark 1-D problem to compare different numerical methods. Variations of this problem are also in common use $[15 ; 16 ; 21 ; 28 ; 56]$. Here we use this test in 2-D for two different materials. The problem domain is a 1 by 1 square. The initial front is a circle with radius 0.25 centered at $(0.5,0.5)$. The gas inside the circle has higher pressure and density (Table 5), causing an outward shock wave and inward rarefaction wave. The material discontinuity is tracked by our front tracking method. With CFL number 0.5 and solid wall boundary condition, simulations are done for five resolutions up to $t=15$, before the shock wave reaches the domain boundary 


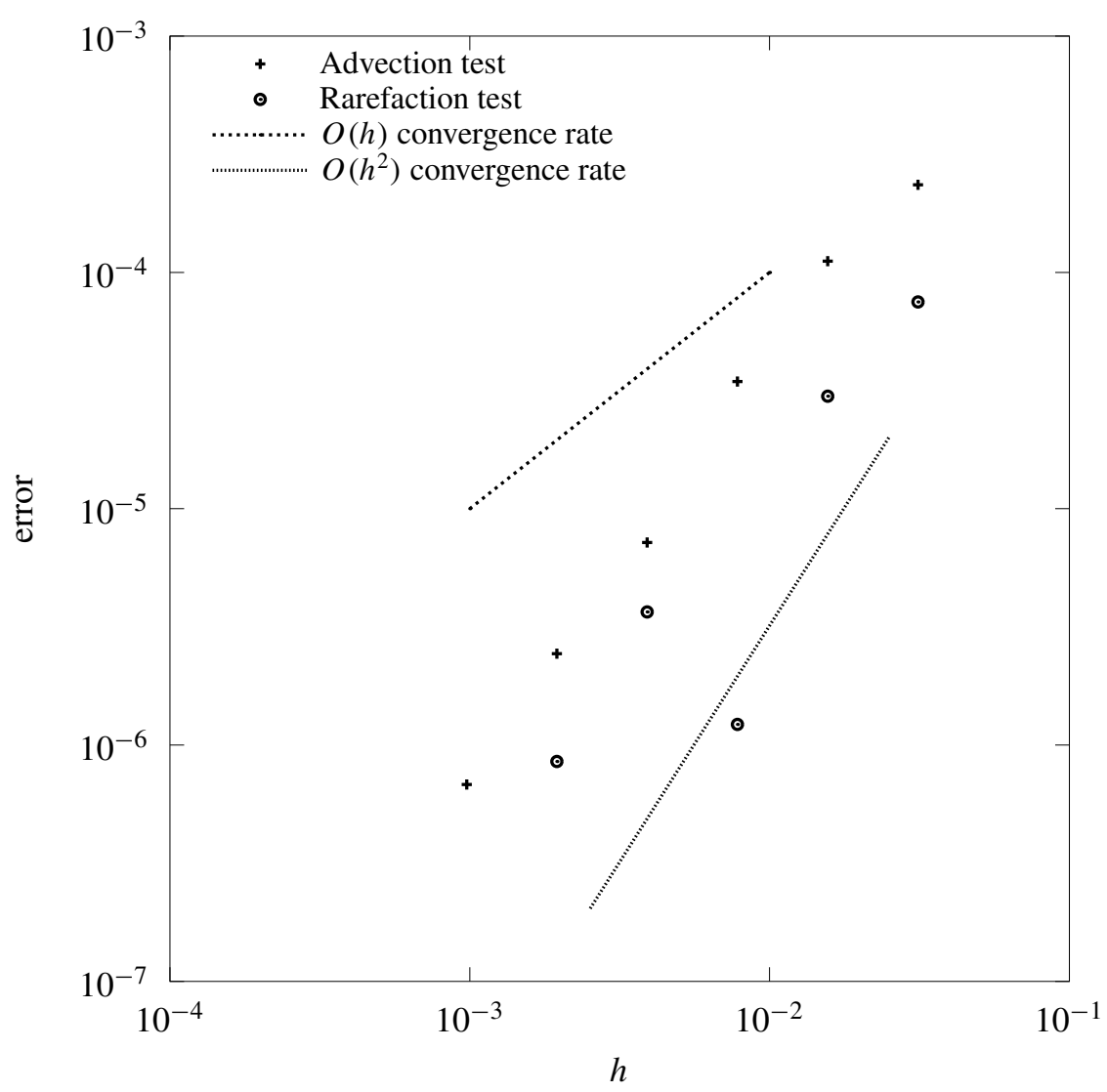

Figure 7. Convergence rate for front position.

(Figure 9), and error and convergence rates are calculated (Table 6). First-order convergence is observed, as expected for a problem containing captured shocks.

5.4. Shock test-shock/front interaction. We chose this problem to observe the nontrivial 2-D interaction of a shock wave with a tracked contact discontinuity. The domain is a [2, 0.5] channel. We use the Sod shock tube problem [53] to initialize the shock wave in gas 1 at $x=1$. The initial material interface $\mathcal{F}(0)$ is a sine wave $\eta_{0} \sin (\omega y+\theta)+x_{0}$ at $x_{0}=1.5$. The wavelength is the same as the width of the channel $\lambda=0.5$, which gives $\omega=2 \pi / \lambda=4 \pi$. The interface amplitude is set to $\eta_{0}=0.16$ and $\theta=\pi / 2$ (see Figure 10). The initial values for each gas are shown in Table 7. Solid wall and periodic boundary conditions are used for vertical and horizontal boundaries, respectively. The simulation is done up to time $t=0.6$, allowing the shock wave to pass through the interface. Using five grid 


\begin{tabular}{|c|c|c|c|c|c|c|}
\hline \multicolumn{7}{|c|}{$\rho$} \\
\hline $\mathrm{h}$ & $L_{1}$ & rate & $L_{2}$ & rate & $L_{\infty}$ & rate \\
\hline $1 / 32$ & $1.51 \times 10^{-3}$ & & $2.40 \times 10^{-3}$ & & $8.07 \times 10^{-3}$ & \\
\hline $1 / 64$ & $2.18 \times 10^{-4}$ & 2.79 & $2.65 \times 10^{-4}$ & 3.18 & $8.94 \times 10^{-4}$ & 3.17 \\
\hline $1 / 128$ & $3.45 \times 10^{-5}$ & 2.66 & $4.24 \times 10^{-5}$ & 2.64 & $1.64 \times 10^{-4}$ & 2.45 \\
\hline $1 / 256$ & $7.93 \times 10^{-6}$ & 2.12 & $1.05 \times 10^{-5}$ & 2.01 & $6.52 \times 10^{-5}$ & 1.33 \\
\hline $1 / 512$ & $2.03 \times 10^{-6}$ & 1.97 & $2.93 \times 10^{-6}$ & 1.84 & $1.74 \times 10^{-5}$ & 1.90 \\
\hline $1 / 1024$ & $5.26 \times 10^{-7}$ & 1.95 & $7.79 \times 10^{-7}$ & 1.91 & $3.76 \times 10^{-6}$ & 2.21 \\
\hline \multicolumn{7}{|c|}{$\rho u \& \rho v$} \\
\hline $\mathrm{h}$ & $L_{1}$ & rate & $L_{2}$ & rate & $L_{\infty}$ & rate \\
\hline $1 / 32$ & $9.89 \times 10^{-4}$ & & $1.48 \times 10^{-3}$ & & $7.48 \times 10^{-3}$ & \\
\hline $1 / 64$ & $1.69 \times 10^{-4}$ & 2.55 & $2.47 \times 10^{-4}$ & 2.58 & $1.26 \times 10^{-3}$ & 2.57 \\
\hline $1 / 128$ & $2.53 \times 10^{-5}$ & 2.74 & $3.83 \times 10^{-5}$ & 2.69 & $3.37 \times 10^{-4}$ & 1.90 \\
\hline $1 / 256$ & $5.36 \times 10^{-6}$ & 2.24 & $7.64 \times 10^{-6}$ & 2.33 & $1.52 \times 10^{-4}$ & 1.15 \\
\hline $1 / 512$ & $1.39 \times 10^{-6}$ & 1.95 & $1.95 \times 10^{-6}$ & 1.97 & $5.89 \times 10^{-5}$ & 1.37 \\
\hline $1 / 1024$ & $3.66 \times 10^{-7}$ & 1.92 & $5.05 \times 10^{-7}$ & 1.95 & $1.36 \times 10^{-5}$ & 2.11 \\
\hline \multicolumn{7}{|c|}{$E$} \\
\hline $\mathrm{h}$ & $L_{1}$ & rate & $L_{2}$ & rate & $L_{\infty}$ & rate \\
\hline $1 / 32$ & $5.10 \times 10^{-3}$ & & $8.38 \times 10^{-3}$ & & $2.85 \times 10^{-2}$ & \\
\hline $1 / 64$ & $6.96 \times 10^{-4}$ & 2.87 & $8.55 \times 10^{-4}$ & 3.29 & $2.65 \times 10^{-3}$ & 3.43 \\
\hline $1 / 128$ & $1.08 \times 10^{-4}$ & 2.69 & $1.33 \times 10^{-4}$ & 2.68 & $4.60 \times 10^{-4}$ & 2.53 \\
\hline $1 / 256$ & $2.52 \times 10^{-5}$ & 2.10 & $3.47 \times 10^{-5}$ & 1.94 & $2.30 \times 10^{-4}$ & 1.00 \\
\hline $1 / 512$ & $6.55 \times 10^{-6}$ & 1.94 & $9.91 \times 10^{-6}$ & 1.81 & $6.10 \times 10^{-5}$ & 1.91 \\
\hline $1 / 1024$ & $1.72 \times 10^{-6}$ & 1.93 & $2.68 \times 10^{-6}$ & 1.89 & $1.32 \times 10^{-5}$ & 2.21 \\
\hline
\end{tabular}

Table 3. Error and convergence rate for perturbation test with $\gamma_{\text {in }}=1.67$ and $\gamma_{\text {out }}=1.4$.

sizes varying from $1 / 32$ to $1 / 512$, error and convergence rates are calculated (see Table 8). The final result for the finest resolution is shown in Figure 10. Again, first-order convergence is observed as expected for a problem containing captured shocks.

5.5. Deforming interface - Richtmyer-Meshkov instability. To test our algorithm in a case comparable to experiment we simulated the initial growth of RichtmyerMeshkov instability(RMI) that occurs when a sudden acceleration is forced on an interface separating fluids with different densities. It is observed that an initial small perturbation on the interface grows after passage of a shock wave in a wide range of Mach numbers [5]. Such growth has been characterized as having a linear phase 


\begin{tabular}{ccccccc}
\multicolumn{7}{c}{$\rho$} \\
\hline $\mathrm{h}$ & $L_{1}$ & rate & $L_{2}$ & rate & $L_{\infty}$ & rate \\
\hline $1 / 32$ & $1.59 \times 10^{-3}$ & & $2.43 \times 10^{-3}$ & & $1.56 \times 10^{-2}$ & \\
$1 / 64$ & $1.92 \times 10^{-4}$ & 3.05 & $2.73 \times 10^{-4}$ & 3.15 & $1.96 \times 10^{-3}$ & 2.99 \\
$1 / 128$ & $3.13 \times 10^{-5}$ & 2.61 & $4.42 \times 10^{-5}$ & 2.63 & $5.49 \times 10^{-4}$ & 1.83 \\
$1 / 256$ & $6.25 \times 10^{-6}$ & 2.33 & $8.56 \times 10^{-6}$ & 2.37 & $8.61 \times 10^{-5}$ & 2.67 \\
$1 / 512$ & $1.55 \times 10^{-6}$ & 2.01 & $2.18 \times 10^{-6}$ & 1.98 & $2.32 \times 10^{-5}$ & 1.90 \\
$1 / 1024$ & $3.98 \times 10^{-7}$ & 1.96 & $5.68 \times 10^{-7}$ & 1.94 & $5.73 \times 10^{-6}$ & 2.02 \\
\hline & & & $\rho u \& \rho v$ & & & \\
\hline $\mathrm{h}$ & $L_{1}$ & rate & $L_{2}$ & rate & $L_{\infty}$ & rate \\
\hline $1 / 32$ & $7.74 \times 10^{-4}$ & & $1.12 \times 10^{-3}$ & & $5.61 \times 10^{-3}$ & \\
$1 / 64$ & $1.76 \times 10^{-4}$ & 2.14 & $2.50 \times 10^{-4}$ & 2.17 & $2.33 \times 10^{-3}$ & 1.27 \\
$1 / 128$ & $2.57 \times 10^{-5}$ & 2.78 & $4.19 \times 10^{-5}$ & 2.58 & $9.44 \times 10^{-4}$ & 1.30 \\
$1 / 256$ & $5.46 \times 10^{-6}$ & 2.23 & $8.01 \times 10^{-6}$ & 2.39 & $2.54 \times 10^{-4}$ & 1.89 \\
$1 / 512$ & $1.44 \times 10^{-6}$ & 1.92 & $2.03 \times 10^{-6}$ & 1.98 & $4.89 \times 10^{-5}$ & 2.38 \\
$1 / 1024$ & $3.76 \times 10^{-7}$ & 1.94 & $5.41 \times 10^{-7}$ & 1.91 & $1.21 \times 10^{-5}$ & 2.01 \\
\hline & & & $E$ & & & \\
\hline $\mathrm{h}$ & & & & & & \\
\hline $1 / 32$ & $5.54 \times 10^{-3}$ & & $8.54 \times 10^{-3}$ & & $5.51 \times 10^{-2}$ & \\
$1 / 64$ & $6.56 \times 10^{-4}$ & 3.08 & $9.53 \times 10^{-4}$ & 3.16 & $6.93 \times 10^{-3}$ & 2.99 \\
$1 / 128$ & $1.07 \times 10^{-4}$ & 2.62 & $1.53 \times 10^{-4}$ & 2.64 & $1.92 \times 10^{-3}$ & 1.85 \\
$1 / 256$ & $2.09 \times 10^{-5}$ & 2.36 & $2.94 \times 10^{-5}$ & 2.38 & $3.00 \times 10^{-4}$ & 2.68 \\
$1 / 512$ & $5.21 \times 10^{-6}$ & 2.00 & $7.53 \times 10^{-6}$ & 1.96 & $8.29 \times 10^{-5}$ & 1.86 \\
$1 / 1024$ & $1.35 \times 10^{-6}$ & 1.95 & $1.98 \times 10^{-6}$ & 1.93 & $2.17 \times 10^{-5}$ & 1.94 \\
\hline & & & & & &
\end{tabular}

Table 4. Error and convergence rate for perturbation test with $\gamma_{\text {in }}=\gamma_{\text {out }}=1.4$.

\begin{tabular}{ccc}
\hline & Gas 1: inside & Gas 2: outside \\
\hline$\rho$ & 3 & 1 \\
$u$ & 0 & 0 \\
$v$ & 0 & 0 \\
$p$ & 3 & 1 \\
$\gamma$ & 1.4 & 1.276 \\
\hline
\end{tabular}

Table 5. Initial values for the 2-D Sod problem.

at the beginning of the process [49], before the nonlinear evolution of spike and bubble development, and of turbulent mixing. 

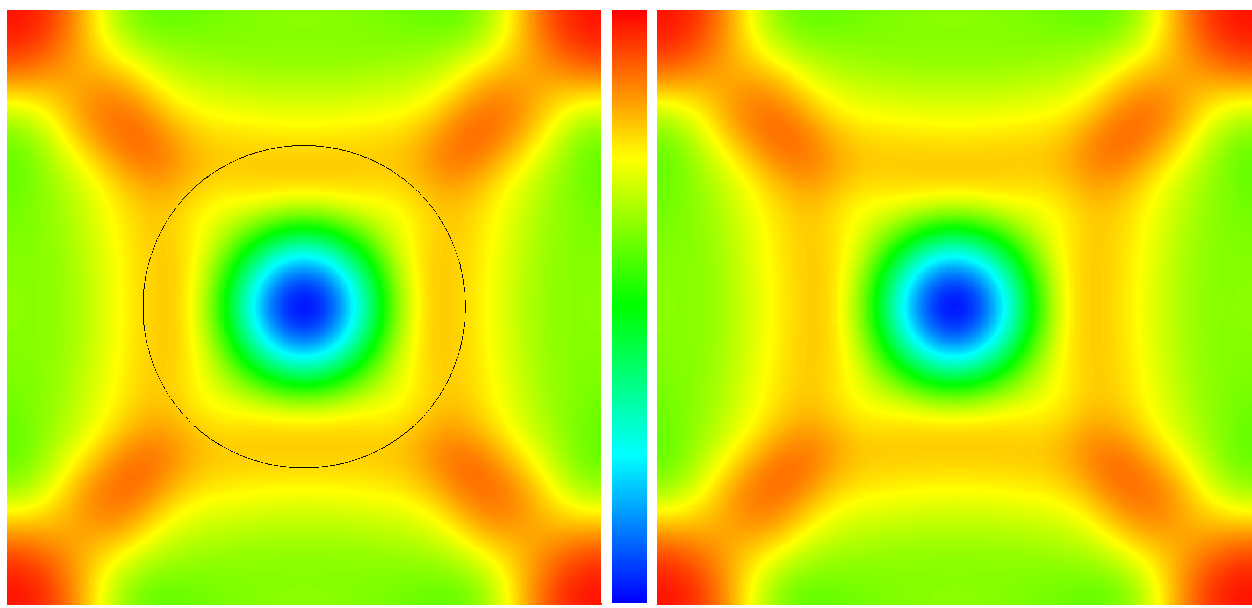

Figure 8. Density profile for smooth perturbation test at $t=0.6$ on a $512 \times 512$ grid. Left: dual gas simulation, front position shown with solid line. Right: result for single gas simulation with no front-tracking. The color table limits are [0.9, 1.04].

\begin{tabular}{ccccccc}
\hline $\mathrm{h}$ & $\rho$ & rate & $\rho u \& \rho v$ & rate & $E$ & rate \\
\hline $1 / 32$ & $2.48 \times 10^{-2}$ & & $1.73 \times 10^{-2}$ & & $8.28 \times 10^{-2}$ & \\
$1 / 64$ & $9.74 \times 10^{-3}$ & 1.35 & $6.49 \times 10^{-3}$ & 1.42 & $3.08 \times 10^{-2}$ & 1.43 \\
$1 / 128$ & $4.91 \times 10^{-3}$ & 0.99 & $3.03 \times 10^{-3}$ & 1.10 & $1.44 \times 10^{-2}$ & 1.09 \\
$1 / 256$ & $2.40 \times 10^{-3}$ & 1.03 & $1.46 \times 10^{-3}$ & 1.05 & $6.90 \times 10^{-3}$ & 1.07 \\
\hline
\end{tabular}

Table 6. Error in $L_{1}$ and convergence rate for the 2-D Sod problem.

\begin{tabular}{cccc}
\hline & Gas 1: postshock & Gas 1: preshock & Gas 2 \\
\hline$\rho$ & 3 & 1 & 1 \\
$u$ & 0 & 0 & 0 \\
$v$ & 0 & 0 & 0 \\
$p$ & 3 & 1 & 1 \\
$\gamma$ & 1.4 & 1.4 & 1.276 \\
\hline
\end{tabular}

Table 7. Initial values for the shock channel problem.

Numerous experimental $[12 ; 26 ; 39 ; 58]$ and numerical studies $[23 ; 27 ; 33$; 55] have been conducted on RMI. Here, we set up the simulation to model an experiment done by Collins and Jacobs [12]. The domain for this simulation is a $[23.73 \mathrm{~cm}, 5.93 \mathrm{~cm}]$ channel. The initial shock is located at $x=10 \mathrm{~cm}$ and the interface is placed at $x=12 \mathrm{~cm}$ with a preshock sinusoidal perturbation of amplitude $a_{0}^{-}=0.18 \mathrm{~cm}$ and wavelength $\lambda=5.93 \mathrm{~cm}$. The initial condition for gas 1 (preshock) and gas 2 are picked to be comparable to the cited experiment, and gas 

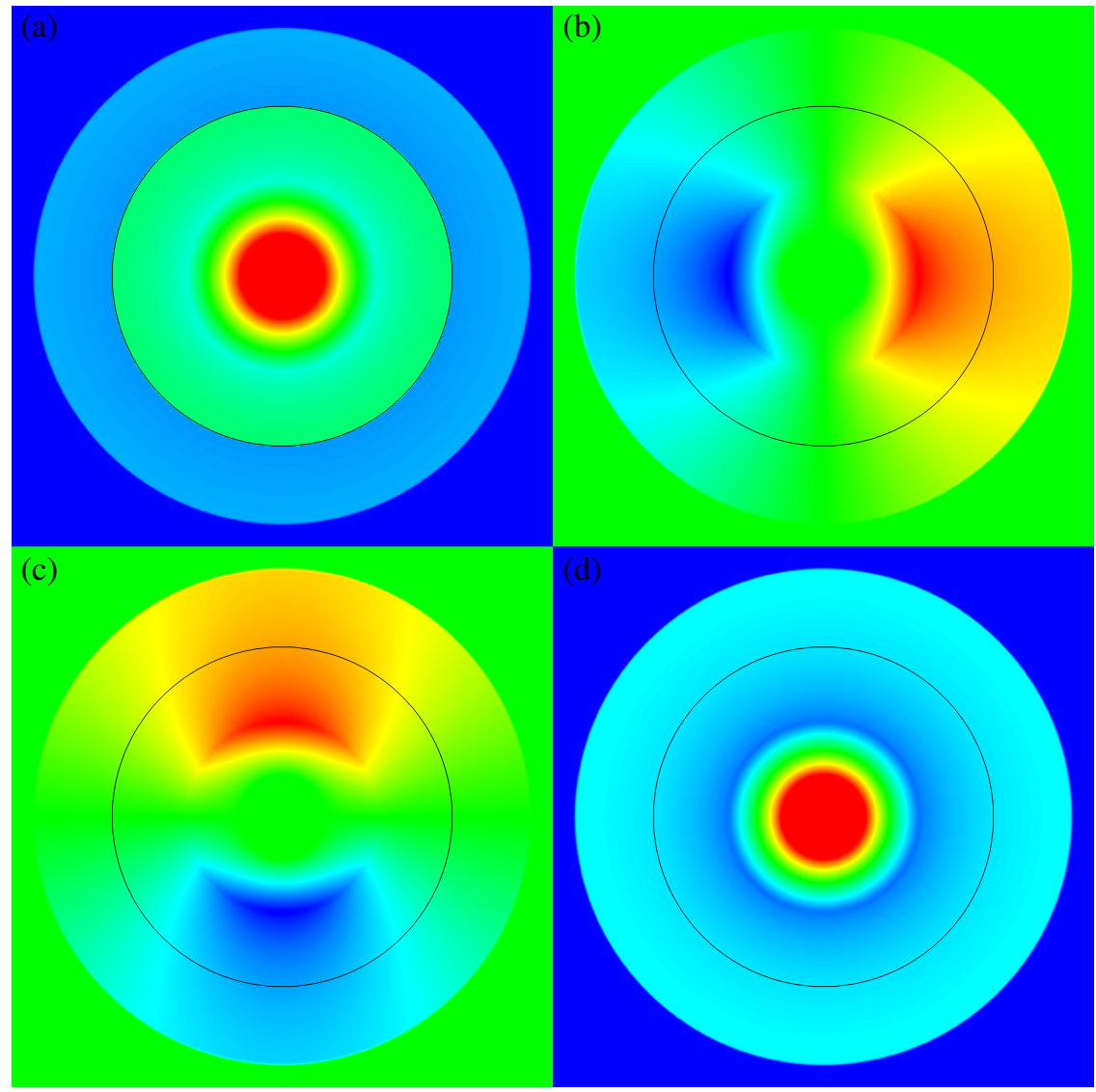

Figure 9. Results for 2-D Sod problem at finest resolution. (a) Density, (b) $x$-momentum, (c) $y$-momentum and (d) pressure. The same color table as Figure 8 is used with limits $[1.0,3.0]$ for density and pressure and $[-0.614,0.614]$ for momentum.

1 (postshock) initial conditions were chosen based on the Rankine-Hugoniot jump condition to have a shock with $\mathrm{Ma}=1.21$ in gas 1 (Table 9). With CFL number 0.5 we simulated the growth of perturbation up to time $0.4 \mathrm{~ms}$ (see Figure 11) and measured the convergence rate of the method (see Table 10).

Richtmyer [49] derived the impulsive growth rate relation for the amplitude of the perturbation based on the linear theory which describes the development of the instability after refraction of shock while the perturbation is small enough to be considered in the linear regime of the process,

$$
\frac{\mathrm{d} \eta}{\mathrm{d} t}=k A^{+} \Delta V_{f} \eta_{0}^{+},
$$

where $k$ is the wavenumber of the perturbation, $A^{+}$is the postshock Atwood number, $\Delta V_{f}$ is the velocity jump on the front following the shock refraction and $\eta_{0}^{+}$is the 


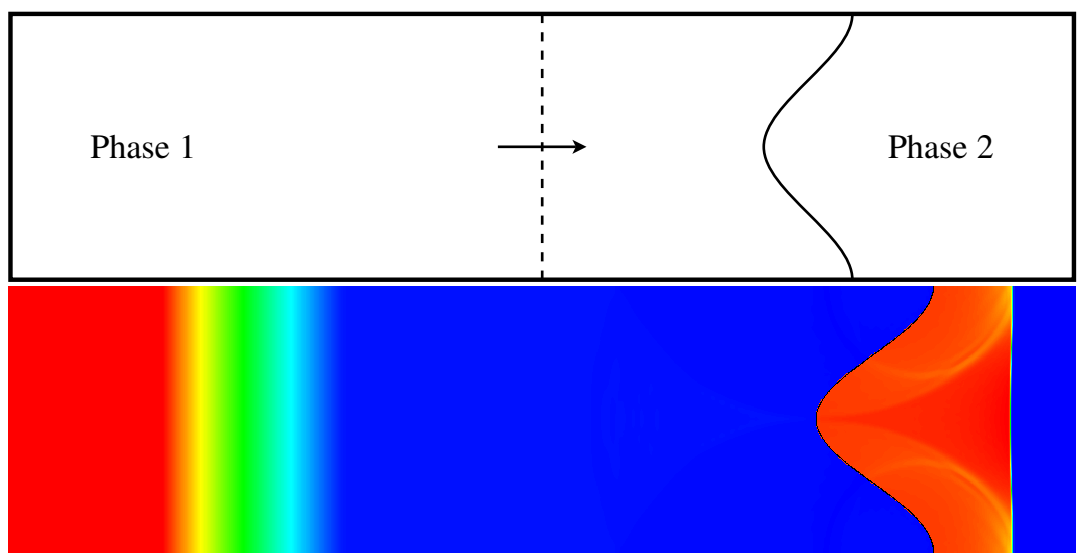

Figure 10. Shock channel initial configuration (top). Pressure profile at $t=0.6$. The same color table as Figure 8 is used with limits [1.67, 3.0] for gas 1 and [1.0, 1.72] for gas 2 (bottom).

\begin{tabular}{ccccc}
\hline $\mathrm{h}$ & $\rho$ & rate & $\rho u$ & rate \\
\hline $1 / 32$ & $8.91 \times 10^{-3}$ & & $8.24 \times 10^{-3}$ & \\
$1 / 64$ & $4.02 \times 10^{-3}$ & 1.15 & $3.63 \times 10^{-3}$ & 1.18 \\
$1 / 128$ & $2.17 \times 10^{-3}$ & 0.88 & $1.89 \times 10^{-3}$ & 0.94 \\
$1 / 256$ & $1.16 \times 10^{-3}$ & 0.91 & $9.82 \times 10^{-4}$ & 0.95 \\
\hline $\mathrm{h}$ & $\rho v$ & rate & $E$ & rate \\
\hline $1 / 32$ & $1.87 \times 10^{-3}$ & & $2.50 \times 10^{-2}$ & \\
$1 / 64$ & $9.09 \times 10^{-4}$ & 1.04 & $1.08 \times 10^{-2}$ & 1.22 \\
$1 / 128$ & $5.21 \times 10^{-4}$ & 0.80 & $5.56 \times 10^{-3}$ & 0.95 \\
$1 / 256$ & $2.77 \times 10^{-4}$ & 0.91 & $2.77 \times 10^{-3}$ & 1.00
\end{tabular}

Table 8. Error in $L_{1}$ and convergence rate for the shock channel problem.

postshock initial perturbation amplitude. For our simulation we have $k=2 \pi / \lambda$, $\rho_{1}^{+}=2.07 \times 10^{-3}, \rho_{2}^{+}=9.05 \times 10^{-3}, A^{+}=\left(\rho_{2}^{+}-\rho_{1}^{+}\right) /\left(\rho_{2}^{+}+\rho_{1}^{+}\right)=0.63$, $\Delta V_{f}=6356.24$, and $\eta_{0}^{+}=0.15$ in CGS units. The calculated amplitude growth using Equation (63) is $\mathrm{d} \eta / \mathrm{d} t=624.82 \mathrm{~cm} / \mathrm{s}$.

From the simulation results we plotted the amplitude of the interface in its linear regime (Figure 12). A linear fit shows a growth rate of $\mathrm{d} \eta / \mathrm{d} t=606.64 \mathrm{~cm} / \mathrm{s}$, while the amplitude growth rate from [12] is $628.64 \mathrm{~cm} / \mathrm{s}$. The simulation results show a good match $(\approx 3 \%$ difference) with experimental and analytical results.

After the initial linear regime of the RMI test, where crests and troughs are symmetric, the interface grows nonlinearly. It becomes visible by the appearance of a bubble and spike, followed by the spike rolling up. The RMI simulation 


\begin{tabular}{lccc}
\hline & Gas 1: postshock & Gas 1: preshock & Gas 2 \\
\hline$\rho\left(\mathrm{g} / \mathrm{cm}^{3}\right)$ & $1.872 \times 10^{-3}$ & $1.351 \times 10^{-3}$ & $5.494 \times 10^{-3}$ \\
$u(\mathrm{~cm} / \mathrm{s})$ & $1.013 \times 10^{4}$ & 0 & 0 \\
$v(\mathrm{~cm} / \mathrm{s})$ & 0 & 0 & 0 \\
$p\left(\mathrm{~g} /\left(\mathrm{cm} . \mathrm{s}^{2}\right)\right)$ & $1.453 \times 10^{6}$ & $9.650 \times 10^{4}$ & $9.650 \times 10^{4}$ \\
$\gamma$ & 1.276 & 1.276 & 1.4 \\
\hline
\end{tabular}

Table 9. Initial values for the RMI problem.

\begin{tabular}{ccccc}
\hline$N_{x}$ & $\rho$ & rate & $\rho u$ & rate \\
\hline 128 & $8.81 \times 10^{-6}$ & & $1.49 \times 10^{-1}$ & \\
256 & $3.91 \times 10^{-6}$ & 1.17 & $6.62 \times 10^{-2}$ & 1.17 \\
512 & $3.05 \times 10^{-6}$ & 0.36 & $5.19 \times 10^{-2}$ & 0.35 \\
1024 & $1.27 \times 10^{-6}$ & 1.27 & $2.64 \times 10^{-2}$ & 1.08 \\
\hline$N_{x}$ & $\rho v$ & rate & $E$ & rate \\
\hline 128 & $2.69 \times 10^{-2}$ & & $1.83 \times 10^{4}$ & \\
256 & $1.13 \times 10^{-2}$ & 1.25 & $8.26 \times 10^{3}$ & 1.15 \\
512 & $5.90 \times 10^{-3}$ & 0.94 & $6.63 \times 10^{3}$ & 0.32 \\
1024 & $3.65 \times 10^{-3}$ & 0.70 & $2.87 \times 10^{3}$ & 1.21 \\
\hline
\end{tabular}

Table 10. Error in $L_{1}$ and convergence rate for RMI problem. $N_{x}$ is the number of cells in the $x$ direction.

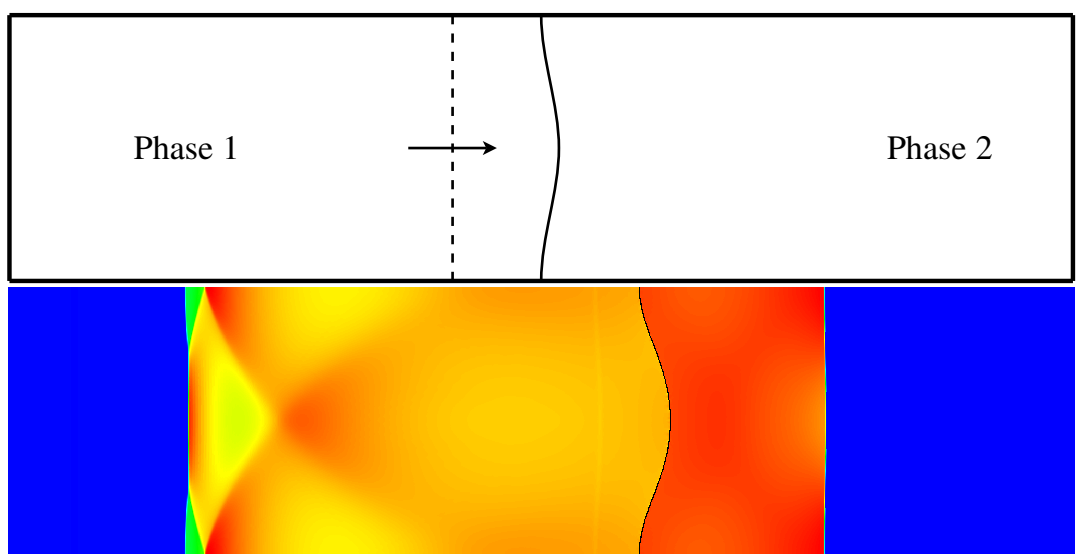

Figure 11. RMI problem initial configuration (top). Pressure profile at $t=0.4 \mathrm{~ms}$. The same color table as Figure 8 is used with limits $[1.87,2.11] \times 10^{-3}\left(\mathrm{~g} / \mathrm{cm}^{3}\right)$ for gas 1 and $[5.49,9.28] \times 10^{-3}\left(\mathrm{~g} / \mathrm{cm}^{3}\right)$ for gas 2 (bottom).

is continued to time $t=2.8 \mathrm{~ms}$ to observe the nonlinear evolution of the front (Figure 13). 


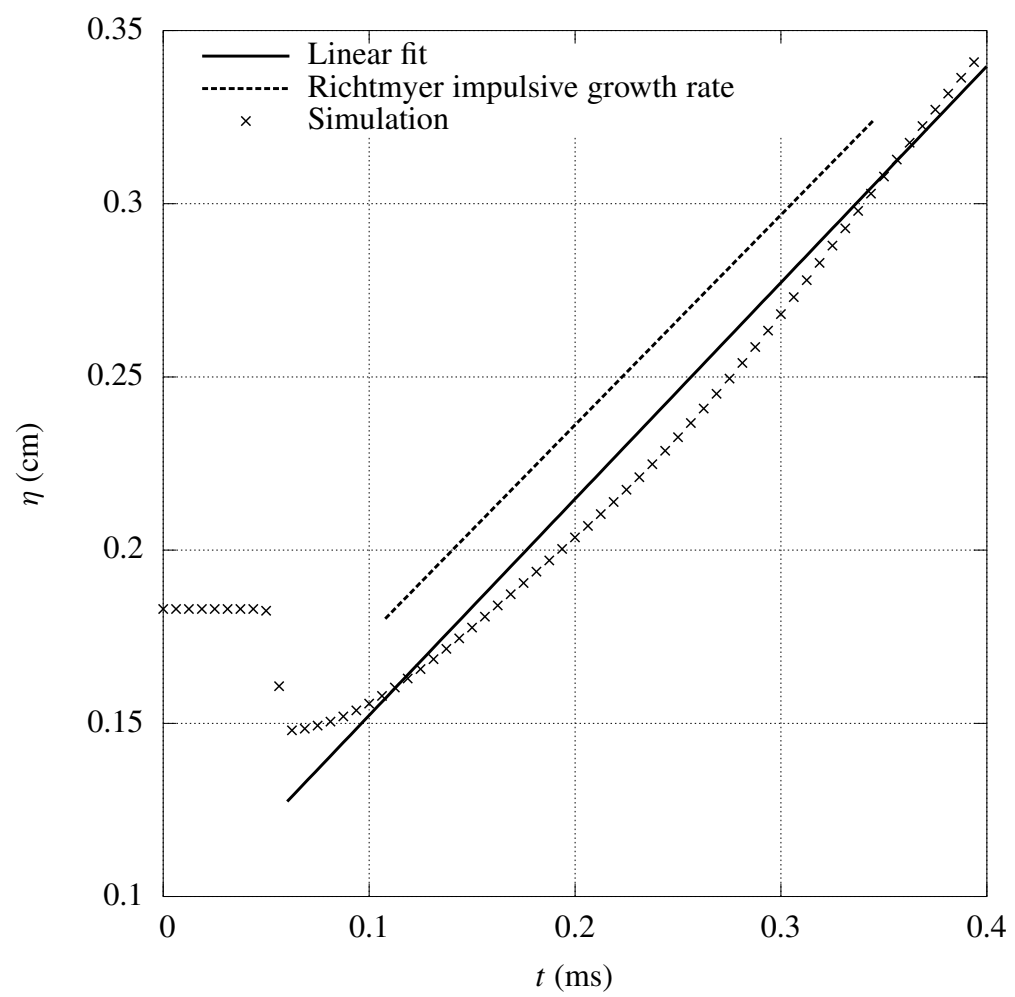

Figure 12. Amplitude of the perturbation on the front in the RMI simulation.

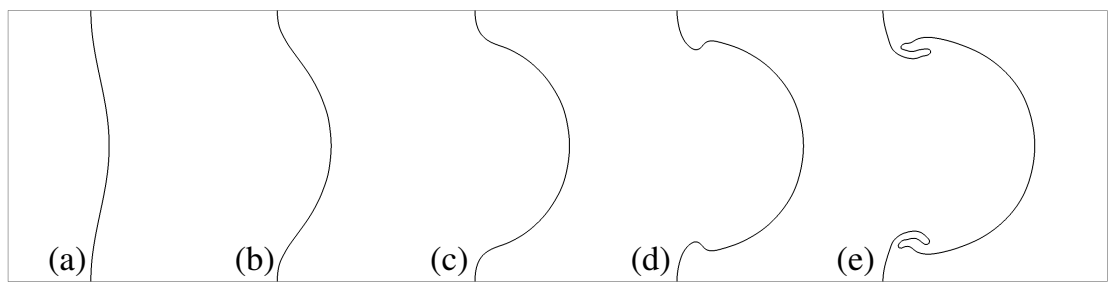

Figure 13. RMI problem front evolution. (a) $t=0$, initial single mode perturbation; (b) $t=0.7 \mathrm{~ms}$, linear growth of perturbation; (c) $t=1.4 \mathrm{~ms}$, asymmetric growth of crests and troughs; (d) $t=2.1 \mathrm{~ms}$, formation of bubble and spike; and (e) $t=2.8 \mathrm{~ms}$, spike roll-up.

5.6. Deforming interface - shock-bubble interaction. The interaction of a shock wave in air with a bubble of helium has become popular for testing multiphase numerical methods. The experimental observations come from Haas and Sturtevant [20], and theoretical and numerical studies include [47; 48]. Numerical studies using this benchmark problem include $[3 ; 15 ; 51 ; 55]$. The initial conditions given in Table 11 induce an incident Mach 1.22 shock (Figure 14). 


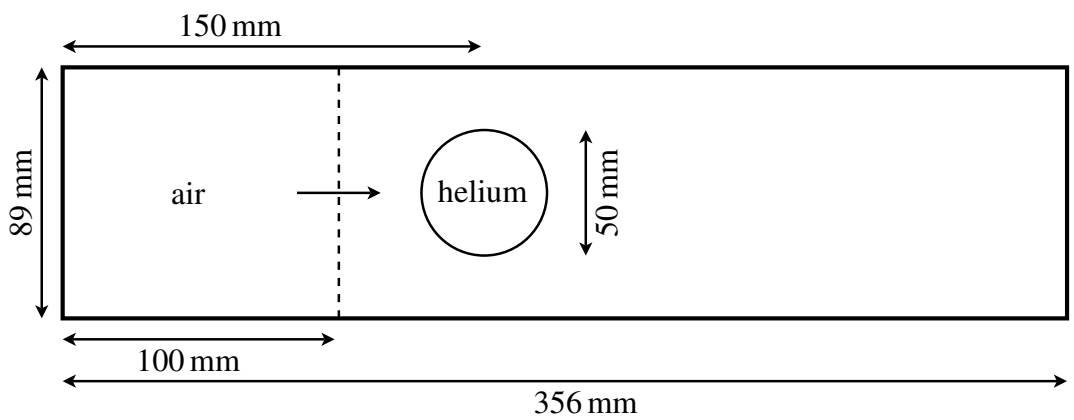

Figure 14. Initial configuration of the shock-bubble problem.

\begin{tabular}{cccc}
\hline & Air: postshock & Air: preshock & Helium \\
\hline$\rho$ & 1.3764 & 1.0 & 0.1819 \\
$u$ & 0.336 & 0 & 0 \\
$v$ & 0 & 0 & 0 \\
$p$ & 1.1213 & 0.7142 & 0.7142 \\
$\gamma$ & 1.4 & 1.4 & 1.648 \\
\hline
\end{tabular}

Table 11. Initial values for the shock-bubble problem.

Using a 512 by 128 grid for the physical domain and CFL number 0.3 , the simulation is done to $t=427 \mu \mathrm{s}$. The results are shown in Figure 15. Comparing the density profiles to the experimental work of Haas and Sturtevant [20], and the adaptive mesh simulations of Quirk and Karni [48], our method accurately captures the dynamics of material interface, and the reflected and transmitted waves.

At later times in the RMI and shock-bubble simulations interface roll-ups are visible (spike roll-up in Figure 13e and jet roll-up in Figure 15j). When the roll-up happens on the material interface, the gradient of the level set becomes discontinuous within the $5 \times 5$ stencil of cells used to compute geometric information. The assumptions underlying the geometry algorithms are violated when this occurs so we terminate the simulation. With local grid refinement this loss of accuracy might be postponed. However, in these experiments perturbations grow at all scales so the loss of continuity will always occur.

\section{Conclusion}

We presented a new front-tracking method for contact discontinuities using the finite volume approach on a Cartesian grid. An essential feature of our method is the computation of geometry information to support space-time finite volume quadratures, using a sequence of discrete level sets [30; 36]. The level sets were advected using a velocity derived from interface-normal Riemann problems. 
(a) $t=32 \mu \mathrm{s}$

(b) $t=52 \mu \mathrm{s}$

(c) $t=62 \mu \mathrm{s}$

(d) $t=72 \mu \mathrm{s}$

(e) $t=82 \mu \mathrm{s}$
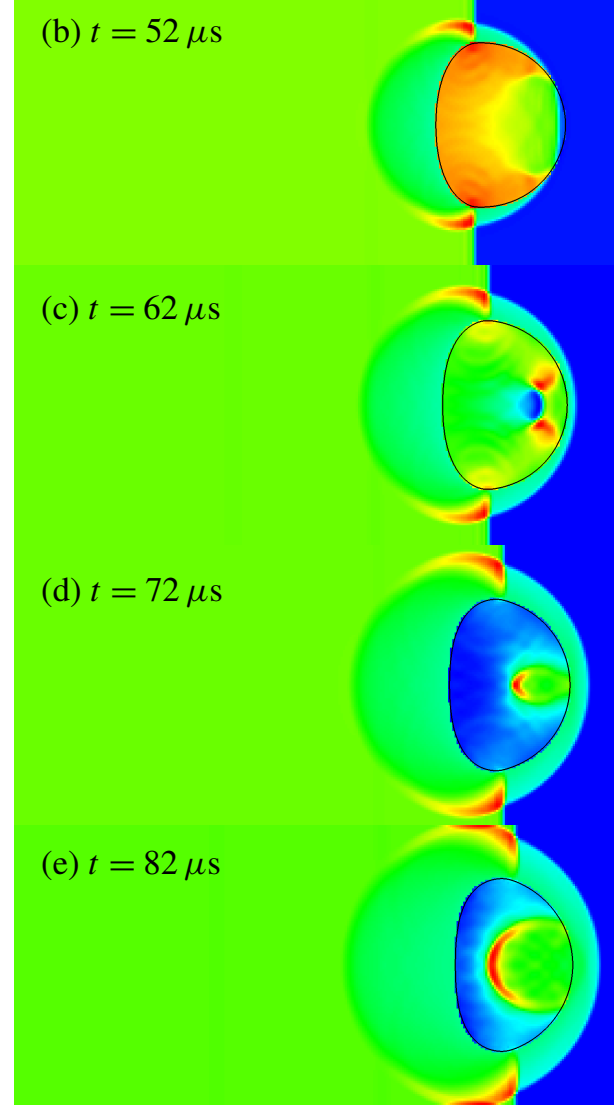

Figure 15. Density profiles of the shock-bubble test. The same color table as Figure 8 is used with limits (a)[0.997, 1.534], (b)[0.988, 1.607], (c)[0.999, 1.625], (d)[1.000, 1.623], and (e)[1.000, 1.646] for air and (a)[0.181, 0.221], (b)[0.178, 0.219], (c)[0.206, 0.221], (d) $[0.215,0.234]$, and (e) $[0.218,0.230]$ for helium.

A variety of convergence tests show that our method is second-order accurate in the $L_{1}, L_{2}$ and $L_{\infty}$ norms, provided there are no shocks present. In the presence of a captured shock the convergence rate reduces to first-order in $L_{1}$.

It should be noted that many parts of the above algorithms may be implemented differently. For example the interpolation and extrapolation algorithms may include limiting operators [45] or different redistribution algorithms may be applied [11]. 


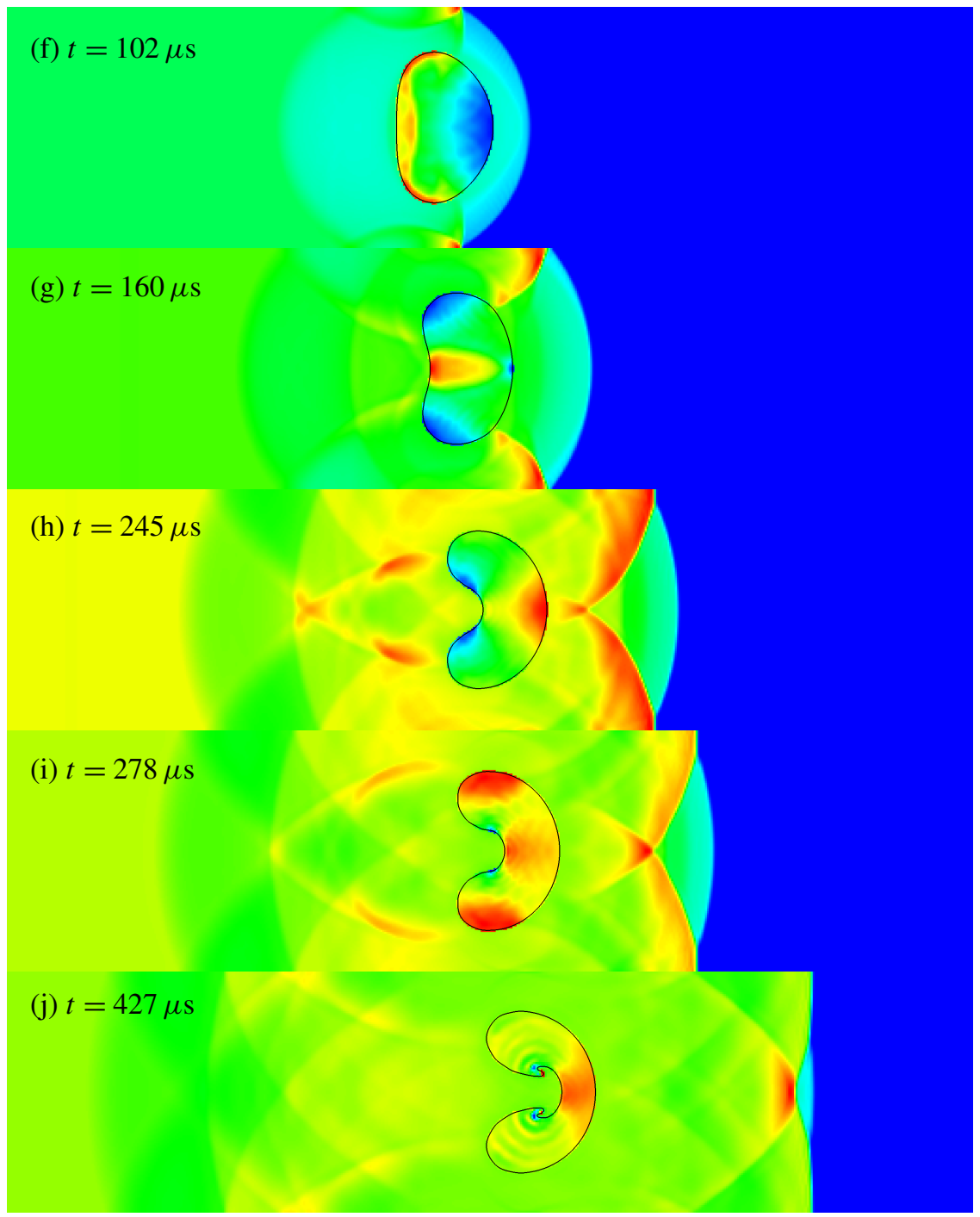

Figure 15. (continued) Density profiles of the shock-bubble test. The same color table as Figure 8 is used with limits (f)[1.000, 1.905], (g)[1.000, 1.664], (h)[1.000, 1.514], (i) $[1.000,1.554]$, and (j) $[1.000,1.584]$ for air and (f)[0.223, 0.231], (g)[0.231, 0.244], (h) $[0.231,0.238]$, (i) $[0.230,0.236]$, and (j) [0.229, 0.247] for helium.

Although such details may vary from case to case, we have shown that a second-order method is achievable if we consider the geometrical information and incorporate such data in the algorithm. While the level set methods we use are well established, there have been many new developments in this field too. Notable developments include the gradient-augmented level set method [40], distance regularized level set 


\begin{tabular}{|c|c|}
\hline Notation & Description \\
\hline$\Upsilon_{i}$ & Cartesian (regular) cell \\
\hline$V_{i, \alpha}^{n}$ & Spatial irregular control volume for gas $\alpha$ at time $n$ in cell $\Upsilon_{i}$ \\
\hline$C_{i, \alpha}$ & Space-time control volume for gas $\alpha$ in $\Upsilon_{i} \times\left[t^{n}, t^{n+1}\right]$ \\
\hline$\left(\vec{x}_{\boldsymbol{i}}, t^{n}\right)$ & Position of a cell center in space and time at time step $n$ \\
\hline$\left(\vec{x}_{\boldsymbol{i}, \alpha}, t^{n}\right)$ & Position of a cell centroid in space and time at time step $n$ for gas $\alpha$ \\
\hline$A_{i \pm \frac{1}{2} e^{d}}$ & $\begin{array}{l}\text { Cartesian (regular) face of } \Upsilon_{i} \times\left[t^{n}, t^{n+1}\right] \text { in lower/higher side in } \\
\text { direction } d\end{array}$ \\
\hline$A_{\boldsymbol{i} \pm \frac{1}{2} \boldsymbol{e}^{d}, \alpha}$ & $\begin{array}{l}\text { Face of } C_{i, \alpha} \text { in lower/higher side in direction } d \text { for gas } \alpha \text { that } \\
\text { coincide with Cartesian grid }\end{array}$ \\
\hline$A_{i}^{f}$ & Front face of $C_{i, \alpha}$ \\
\hline$\left(\vec{x}_{i \pm \frac{1}{2} e^{d}}, t^{n+\frac{1}{2}}\right)$ & Position of a face center in space and time for a regular face \\
\hline$\left(\vec{x}_{\boldsymbol{i} \pm \frac{1}{2} e^{d}, \alpha}, t_{i \pm \frac{1}{2} e^{d}, \alpha}\right)$ & $\begin{array}{l}\text { Position of a face centroid in space and time for a irregular control } \\
\text { volume for gas } \alpha\end{array}$ \\
\hline$\left(\vec{x}_{i}^{f}, t_{i}^{f}\right)$ & Position of a front centroid position in space and time \\
\hline $\boldsymbol{F}_{i \pm \frac{1}{2} e^{d}, \alpha}^{\mathrm{cr}}$ & Flux at face center \\
\hline $\boldsymbol{F}_{i \pm \frac{1}{2} \boldsymbol{e}^{d}, \alpha}^{\mathrm{cd}^{2}}$ & Flux at face centroid \\
\hline $\boldsymbol{F}_{\boldsymbol{i}, d, \alpha}^{f{ }^{\prime}}$ & Flux at front centroid \\
\hline
\end{tabular}

Table 12. Geometrical notation.

evolution [29], and the use of queuing algorithms [59] or hash table data structures for fast marching methods [6].

We validated our method with the simulation of single mode Richtmyer-Meshkov instability and shock-bubble interaction test. Simulation results are in good agreement with theoretical predictions and with experimental results.

\section{Acknowledgements}

This material is based on work supported by the National Science foundation under Grant No. DMS-0810939, and the Institute of International Education.

\section{References}

[1] D. Adalsteinsson and J. A. Sethian, The fast construction of extension velocities in level set methods, J. Comput. Phys. 148 (1999), no. 1, 2-22. MR 99j:65189 Zbl 0919.65074

[2] J. B. Bell, P. Colella, and M. L. Welcome, Conservative front-tracking for inviscid compressible flow, 10-th AIAA Computational Fluid Dynamics Conference (A. D. Vakili and C. Gauthier, eds.), 1991, pp. 814-822. 
[3] J. Bell, M. Berger, J. Saltzman, and M. Welcome, Three-dimensional adaptive mesh refinement for hyperbolic conservation laws, SIAM J. Sci. Comput. 15 (1994), no. 1, 127-138. MR 95d:65070 Zbl 0793.65072

[4] M. J. Berger, C. Helzel, and R. J. Leveque, h-box methods for the approximation of hyperbolic conservation laws on irregular grids, SIAM J. Numer. Anal. 41 (2003), no. 3, 893-918. MR 2004g:65103 Zbl 1066.65082

[5] M. Brouillette, The Richtmyer-Meshkov instability, Annual review of fluid mechanics (J. L. Lumley, S. H. Davis, and P. Moin, eds.), no. 34, Annual Reviews, Palo Alto, CA, 2002, pp. 445468. MR 2003c:76059 Zbl 1047.76025

[6] E. Brun, A. Guittet, and F. Gibou, A local level-set method using a hash table data structure, J. Comput. Phys. 231 (2012), no. 6, 2528-2536. MR 2881030 Zbl 1242.65156

[7] I.-L. Chern and P. Colella, A conservative front tracking method for hyperbolic conservation laws, Tech. Report LLNL Report No. UCRL-97200, Lawrence Livermore National Laboratory, 1987.

[8] P. Colella, H. M. Glaz, and R. E. Ferguson, Multifluid algorithms for Eulerian finite difference methods, Tech. report, Lawrence Berkeley National Laboratory, 1996.

[9] P. Colella, Multidimensional upwind methods for hyperbolic conservation laws, J. Comput. Phys. 87 (1990), no. 1, 171-200. MR 91c:76087 Zbl 0694.65041

[10] _ Volume-of-fluid methods for partial differential equations, Godunov methods (E. Toro, ed.), Springer, New York, 2001, pp. 161-177. MR 1963590 Zbl 0989.65118

[11] P. Colella, D. T. Graves, B. J. Keen, and D. Modiano, A Cartesian grid embedded boundary method for hyperbolic conservation laws, J. Comput. Phys. 211 (2006), no. 1, 347-366. MR 2006i:65142 Zbl 1120.65324

[12] B. D. Collins and J. W. Jacobs, PLIF flow visualization and measurements of the RichtmyerMeshkov instability of an air/SF6 interface, J. Fluid Mech. 464 (2002), 113-136.

[13] J. Du, B. Fix, J. Glimm, X. Jia, X. Li, Y. Li, and L. Wu, A simple package for front tracking, J. Comput. Phys. 213 (2006), no. 2, 613-628. MR 2006j:65306 Zbl 1089.65128

[14] A. du Chéné, C. Min, and F. Gibou, Second-order accurate computation of curvatures in a level set framework using novel high-order reinitialization schemes, J. Sci. Comput. 35 (2008), no. 2-3, 114-131. MR 2009e:65038 Zbl 1203.65043

[15] R. P. Fedkiw, T. Aslam, B. Merriman, and S. Osher, A non-oscillatory Eulerian approach to interfaces in multimaterial flows (the ghost fluid method), J. Comput. Phys. 152 (1999), no. 2, 457-492. MR 2000c:76061 Zbl 0957.76052

[16] C. Gatti-Bono, P. Colella, and D. Trebotich, A second-order accurate conservative fronttracking method in one dimension, SIAM J. Sci. Comput. 31 (2009/10), no. 6, 4795-4813. MR 2011k:65118 Zbl 05834168

[17] J. Glimm, E. Isaacson, D. Marchesin, and O. McBryan, Front tracking for hyperbolic systems, Adv. in Appl. Math. 2 (1981), no. 1, 91-119. MR 82i:76097 Zbl 0459.76069

[18] J. Glimm, X. L. Li, Y. Liu, and N. Zhao, Conservative front tracking and level set algorithms, Proc. Natl. Acad. Sci. USA 98 (2001), no. 25, 14198-14201. MR 1867514 Zbl 1005.65091

[19] J. Glimm, Y. Liu, Z. Xu, and N. Zhao, Conservative front tracking with improved accuracy, SIAM J. Numer. Anal. 41 (2003), no. 5, 1926-1947. MR 2004j:76109 Zbl 1053.35093

[20] J.-F. Haas and B. Sturtevant, Interaction of weak shock waves with cylindrical and spherical gas inhomogeneities, J. Fluid Mech. 181 (1987), 41-76. 
[21] A. Harten, High resolution schemes for hyperbolic conservation laws, J. Comput. Phys. 49 (1983), no. 3, 357-393. MR 84g:65115 Zbl 0565.65050

[22] C. W. Hirt and B. D. Nichols, Volume of fluid (VOF) method for the dynamics of free boundaries, J. Comput. Phys. 39 (1981), no. 1, 201-225.

[23] R. L. Holmes, G. Dimonte, B. Fryxell, M. L. Gittings, J. W. Grove, M. Schneider, D. H. Sharp, A. L. Velikovich, R. P. Weaver, and Q. Zhang, Richtmyer-Meshkov instability growth: experiment, simulation and theory, J. Fluid Mech. 389 (1999), 55-79. MR 1707239 Zbl 0954.76026

[24] J. M. Hyman, Numerical methods for tracking interfaces, Physica D: Nonlinear Phenomena 12 (1984), no. 1-3, 396-407. Zbl 0604.65092

[25] G.-S. Jiang and D. Peng, Weighted ENO schemes for Hamilton-Jacobi equations, SIAM J. Sci. Comput. 21 (2000), no. 6, 2126-2143. MR 2001e:65124 Zbl 0957.35014

[26] M. A. Jones and J. W. Jacobs, A membraneless experiment for the study of Richtmyer-Meshkov instability of a shock-accelerated gas interface, Physics of Fluids 9 (1997), no. 10, 3078-3085.

[27] M. Latini, O. Schilling, and W. S. Don, High-resolution simulations and modeling of reshocked single-mode Richtmyer-Meshkov instability: Comparison to experimental data and to amplitude growth model predictions, Physics of Fluids 19 (2007), no. 2, 024104. Zbl 1146.76456

[28] R. J. LeVeque, Finite volume methods for hyperbolic problems, Cambridge University Press, 2002. MR 2003h:65001 Zbl 1010.65040

[29] C. Li, C. Xu, C. Gui, and M. D. Fox, Distance regularized level set evolution and its application to image segmentation, IEEE Trans. Image Process. 19 (2010), no. 12, 3243-3254. MR 2011m:94019

[30] T. J. Ligocki, P. O. Schwartz, J. Percelay, and P. Colella, Embedded boundary grid generation using the divergence theorem, implicit functions, and constructive solid geometry, Journal of Physics: Conference Series 125 (2008), no. 1, 012080.

[31] R. Malladi, J. Sethian, and B. Vemuri, Shape modeling with front propagation: a level set approach, IEEE Trans. Pattern Anal. and Mach. Intel. 17 (1995), no. 2, 158-175.

[32] R. Menikoff and B. J. Plohr, The Riemann problem for fluid flow of real materials, Rev. Modern Phys. 61 (1989), no. 1, 75-130. MR 90a:35142 Zbl 1129.35439

[33] K. O. Mikaelian, Numerical simulations of Richtmyer-Meshkov instabilities in finite-thickness fluid layers, Physics of Fluids 8 (1996), no. 5, 1269-1292. Zbl 1086.76018

[34] G. Miller, Numerical analysis for engineers and scientists, Cambridge University Press, 2014. MR 3290285 Zbl 1294.00002

[35] G. H. Miller and P. Colella, A conservative three-dimensional Eulerian method for coupled solid-fluid shock capturing, J. Comput. Phys. 183 (2002), no. 1, 26-82. MR 2003j:76080 Zbl 1057.76558

[36] G. H. Miller and D. Trebotich, An embedded boundary method for the Navier-Stokes equations on a time-dependent domain, Commun. Appl. Math. Comput. Sci. 7 (2012), no. 1, 1-31. MR 2893419 Zbl 1273.35215

[37] __ A front tracking embedded boundary method for two-fluid incompressible NavierStokes problems with surface tension, preprint, 2014, submitted.

[38] G. H. Miller and E. G. Puckett, A high-order Godunov method for multiple condensed phases, J. Comput. Phys. 128 (1996), no. 1, 134-164. Zbl 0861.65117

[39] B. Motl, J. Oakley, D. Ranjan, C. Weber, M. Anderson, and R. Bonazza, Experimental validation of a Richtmyer-Meshkov scaling law over large density ratio and shock strength ranges, Physics of Fluids 21 (2009), no. 12, 126102. Zbl 1183.76364 
[40] J.-C. Nave, R. R. Rosales, and B. Seibold, A gradient-augmented level set method with an optimally local, coherent advection scheme, J. Comput. Phys. 229 (2010), no. 10, 3802-3827. MR 2011a:65256 Zbl 1189.65214

[41] W. Noh and P. Woodward, SLIC (simple line interface calculation), Proceedings of the fifth international conference on numerical methods in fluid dynamics (A. I. van de Vooren and P. J. Zandbergen, eds.), Lecture Notes in Physics, no. 59, Springer, Berlin, 1976, pp. 330-340.

[42] R. R. Nourgaliev, M.-S. Liou, and T. G. Theofanous, Numerical prediction of interfacial instabilities: sharp interface method (SIM), J. Comput. Phys. 227 (2008), no. 8, 3940-3970. MR 2009d:76054 Zbl 1275.76164

[43] S. Osher and R. Fedkiw, Level set methods and dynamic implicit surfaces, Applied Mathematical Sciences, no. 153, Springer, New York, 2003. MR 2003j:65002 Zbl 1026.76001

[44] S. Osher and J. A. Sethian, Fronts propagating with curvature-dependent speed: algorithms based on Hamilton-Jacobi formulations, J. Comput. Phys. 79 (1988), no. 1, 12-49. MR 89h: 80012 Zbl 0659.65132

[45] R. B. Pember, J. B. Bell, P. Colella, W. Y. Crutchfield, and M. L. Welcome, An adaptive Cartesian grid method for unsteady compressible flow in irregular regions, J. Comput. Phys. 120 (1995), no. 2, 278-304. MR 96d:76081 Zbl 0842.76056

[46] D. Peng, B. Merriman, S. Osher, H. Zhao, and M. Kang, A PDE-based fast local level set method, J. Comput. Phys. 155 (1999), no. 2, 410-438. MR 2000j:65104 Zbl 0964.76069

[47] J. M. Picone and J. P. Boris, Vorticity generation by shock propagation through bubbles in a gas, J. Fluid Mech. 189 (1988), 23-51.

[48] J. J. Quirk and S. Karni, On the dynamics of a shock-bubble interaction, J. Fluid Mech. 318 (1996), 129-163. Zbl 0877.76046

[49] R. D. Richtmyer, Taylor instability in shock acceleration of compressible fluids, Comm. Pure Appl. Math. 13 (1960), 297-319. MR 22 \#5275

[50] V. Sabelnikov, A. Y. Ovsyannikov, and M. Gorokhovski, Modified level set equation and its numerical assessment, J. Comput. Phys. 278 (2014), 1-30. MR 3261080

[51] R. Saurel and R. Abgrall, A simple method for compressible multifluid flows, SIAM J. Sci. Comput. 21 (1999), no. 3, 1115-1145. MR 2001b:65089 Zbl 0957.76057

[52] J. A. Sethian, Level set methods and fast marching methods, 2-nd ed., Cambridge Monographs on Applied and Computational Mathematics, no. 3, Cambridge University Press, 1999. MR 2000c:65015 Zbl 0973.76003

[53] G. A. Sod, A survey of several finite difference methods for systems of nonlinear hyperbolic conservation laws, J. Comput. Phys. 27 (1978), no. 1, 1-31. MR 58 \#13770 Zbl 0387.76063

[54] M. Sussman, P. Smereka, and S. Osher, A level set approach for computing solutions to incompressible two-phase flow, J. Comput. Phys. 114 (1994), no. 1, 146-159. Zbl 0808.76077

[55] H. Terashima and G. Tryggvason, A front-tracking/ghost-fluid method for fluid interfaces in compressible flows, J. Comput. Phys. 228 (2009), no. 11, 4012-4037. Zbl 1171.76046

[56] E. F. Toro, Riemann solvers and numerical methods for fluid dynamics, 2-nd ed., Springer, Berlin, 1999. MR 2000f:76091 Zbl 0923.76004

[57] M. Vahab, A front-tracking shock-capturing method for two fluids, Ph.D. thesis, University of California, Davis, 2014, p. 63. MR 3295245

[58] M. Vetter and B. Sturtevant, Experiments on the Richtmyer-Meshkov instability of an air/SF 6 interface, Shock Waves 4 (1995), no. 5, 247-252. 
[59] L. Yatziv, A. Bartesaghi, and G. Sapiro, $O(N)$ implementation of the fast marching algorithm, J. Comput. Phys. 212 (2006), no. 2, 393-399. Zbl 1083.65083

[60] H.-K. Zhao, T. Chan, B. Merriman, and S. Osher, A variational level set approach to multiphase motion, J. Comput. Phys. 127 (1996), no. 1, 179-195. MR 97g:80013 Zbl 0957.76057

Received December 26, 2013. Revised April 22, 2015.

MEHDI VAHAB: mvahab@f su.edu

Department of Mathematics, Florida State University, Tallahassee, FL 32306

GREGORY H. MILLER: grgmiller@ucdavis .edu

Department of Chemical Engineering and Materials Science, University of California, Davis, 1 Shields Avenue, Davis, CA 95616, United States 


\title{
Communications in Applied Mathematics and Computational Science
}

\author{
msp.org/camcos
}

EDITORS

MANAGING EDITOR

John B. Bell

Lawrence Berkeley National Laboratory, USA

jbbell@lbl.gov

\section{BOARD OF EDITORS}

\begin{tabular}{|c|c|c|c|}
\hline Marsha Berger & $\begin{array}{l}\text { New York University } \\
\text { berger@cs.nyu.edu }\end{array}$ & Ahmed Ghoniem & $\begin{array}{l}\text { Massachusetts Inst. of Technology, USA } \\
\text { ghoniem@mit.edu }\end{array}$ \\
\hline Alexandre Chorin & $\begin{array}{l}\text { University of California, Berkeley, USA } \\
\text { chorin@math.berkeley.edu }\end{array}$ & Raz Kupferman & $\begin{array}{l}\text { The Hebrew University, Israel } \\
\text { raz@math.huji.ac.il }\end{array}$ \\
\hline Phil Colella & $\begin{array}{l}\text { Lawrence Berkeley Nat. Lab., USA } \\
\text { pcolella@lbl.gov }\end{array}$ & Randall J. LeVeque & $\begin{array}{l}\text { University of Washington, USA } \\
\text { rj1@amath.washington.edu }\end{array}$ \\
\hline Peter Constantin & $\begin{array}{l}\text { University of Chicago, USA } \\
\text { const@cs.uchicago.edu }\end{array}$ & Mitchell Luskin & $\begin{array}{l}\text { University of Minnesota, USA } \\
\text { luskin@umn.edu }\end{array}$ \\
\hline Maksymilian Dryja & $\begin{array}{l}\text { Warsaw University, Poland } \\
\text { maksymilian.dryja@acn.waw.pl }\end{array}$ & Yvon Maday & $\begin{array}{l}\text { Université Pierre et Marie Curie, France } \\
\text { maday@ann.jussieu.fr }\end{array}$ \\
\hline M. Gregory Forest & $\begin{array}{l}\text { University of North Carolina, USA } \\
\text { forest@amath.unc.edu }\end{array}$ & James Sethian & $\begin{array}{l}\text { University of California, Berkeley, USA } \\
\text { sethian@ math.berkeley.edu }\end{array}$ \\
\hline Leslie Greengard & $\begin{array}{l}\text { New York University, USA } \\
\text { greengard@cims.nyu.edu }\end{array}$ & Juan Luis Vázquez & $\begin{array}{l}\text { Universidad Autónoma de Madrid, Spain } \\
\text { juanluis.vazquez@uam.es }\end{array}$ \\
\hline Rupert Klein & $\begin{array}{l}\text { Freie Universität Berlin, Germany } \\
\text { rupert.klein@pik-potsdam.de }\end{array}$ & Alfio Quarteroni & $\begin{array}{l}\text { Ecole Polytech. Féd. Lausanne, Switzerland } \\
\text { alfio.quarteroni@epfl.ch }\end{array}$ \\
\hline \multirow[t]{2}{*}{ Nigel Goldenfeld } & $\begin{array}{l}\text { University of Illinois, USA } \\
\text { nigel@uiuc.edu }\end{array}$ & Eitan Tadmor & $\begin{array}{l}\text { University of Maryland, USA } \\
\text { etadmor@cscamm.umd.edu }\end{array}$ \\
\hline & & Denis Talay & $\begin{array}{l}\text { INRIA, France } \\
\text { denis.talay@inria.fr }\end{array}$ \\
\hline
\end{tabular}

\section{PRODUCTION}

production@msp.org

Silvio Levy, Scientific Editor

See inside back cover or msp.org/camcos for submission instructions.

The subscription price for 2016 is US $\$ 95 /$ year for the electronic version, and $\$ 135 /$ year $(+\$ 15$, if shipping outside the US) for print and electronic. Subscriptions, requests for back issues from the last three years and changes of subscribers address should be sent to MSP.

Communications in Applied Mathematics and Computational Science (ISSN 2157-5452 electronic, 1559-3940 printed) at Mathematical Sciences Publishers, 798 Evans Hall \#3840, c/o University of California, Berkeley, CA 94720-3840, is published continuously online. Periodical rate postage paid at Berkeley, CA 94704, and additional mailing offices.

CAMCoS peer review and production are managed by EditFLOW ${ }^{\circledR}$ from MSP.

\section{PUBLISHED BY}

mathematical sciences publishers

nonprofit scientific publishing

http://msp.org/

(C) 2016 Mathematical Sciences Publishers 


\section{Communications in Applied Mathematics and Computational Science}

A front-tracking shock-capturing method for two gases

Mehdi Vahab and Gregory H. Miller

Identifying turbulent structures through topological segmentation

Peer-Timo Bremer, Andrea Gruber, Janine C. Bennett,

Attila Gyulassy, Hemanth Kolla, Jacqueline H. Chen and

RAY W. GROUT

An asymptotic-preserving scheme for systems of conservation laws with source terms on 2D unstructured meshes

Christophe Berthon, Guy Moebs, Céline Sarazin-Desbois and Rodolphe Turpault

An immersed boundary method for rigid bodies

Bakytzhan Kallemov, Amneet Pal Singh Bhalla, Boyce E.

Griffith and Aleksandar DoneV 